\title{
Approximate thermodynamic structure for driven lattice gases in contact
}

\author{
Punyabrata Pradhan, Robert Ramsperger, and Udo Seifert \\ II. Institut für Theoretische Physik, Universität Stuttgart, Stuttgart 70550, Germany
}

\begin{abstract}
For a class of nonequilibrium systems, called driven lattice gases, we study what happens when two systems are kept in contact and allowed to exchange particles with the total number of particles conserved. Both for attractive and repulsive nearest-neighbor interactions among particles and for a wide range of parameter values, we find that, to a good approximation, one could define an intensive thermodynamic variable, like equilibrium chemical potential, which determines the final steady state for two initially separated driven lattice gases brought into contact. However, due to nontrivial contact dynamics, there are also observable deviations from this simple thermodynamic law. To illustrate the role of the contact dynamics, we study a variant of the zero range process and discuss how the deviations could be explained by a modified large deviation principle. We identify an additional contribution to the large deviation function, which we call the excess chemical potential, for the variant of the zero range process as well as the driven lattice gases. The excess chemical potential depends on the specifics of the contact dynamics and is in general a-priori unknown. A contact dependence implies that even though an intensive variable may equalize, the zeroth law could still be violated.
\end{abstract}

PACS numbers: 05.70.Ln, 05.20.-y

\section{INTRODUCTION}

Equilibrium systems, which satisfy detailed balance and therefore do not have any particle or energy current, are based on a well founded thermodynamic theory. Studies of equilibrium systems start with the zeroth law which is the corner-stone of equilibrium thermodynamics. The zeroth law states that, in equilibrium, there exists a set of intensive variables, each of which being conjugate to a corresponding extensive conserved quantity, and these intensive variables equalize when two systems are kept in contact and allowed to exchange the conserved quantities. Specifically, an equilibrium system in contact with a reservoir is characterized by the familiar Boltzmann distribution where the probability of a microstate $C$ is given by $P(C) \sim \exp [-\beta\{H(C)-\mu N\}]$ with $H(C)$ the internal energy of configuration $C, N$ the number of particles in the system, and the intensive variables $\beta$ and $\mu$ being the inverse temperature and the chemical potential of the reservoir, respectively. These variables $\beta$ and $\mu$ are conjugate to energy and particle-number of the system. Moreover, in equilibrium, there is a class of general fluctuation-response relations, collectively called the Fluctuation Dissipation Theorem (FDT), which relate the response of a system upon change of an intensive variable (e.g., chemical potential) to the fluctuation in the corresponding extensive variable (e.g., particle-number).

One could inquire whether there can be a similar thermodynamic structure for nonequilibrium systems as well. Among the vast class of nonequilibrium systems, one ubiquitous subclass is that of systems having nonequilibrium steady state (NESS) [1]. The systems in a NESS have time-independent macroscopic properties which is similar to that of systems in equilibrium. However, unlike in equilibrium, they have a steady current and gener- ally cannot be characterized by the Boltzmann distributions with an a-priori known energy functions. Perhaps not surprisingly, even for this conceptually simplest class of driven systems with NESS, there is no well founded thermodynamic structure. Intensive studies in this direction to find a suitable framework for description of NESS have been undertaken [2 [6]. In this paper, we ask whether a homogeneously driven many-particle system can be characterized in terms of an intensive thermodynamic variable which equalizes for two systems in contact. We address this question using a simple class of stochastic models called driven lattice gases.

Although there have been many attempts to define an intensive variable for driven systems in various specific contexts [ 10], there was no general formulation in this regard until recently when a prescription to define such a variable for driven systems was proposed by invoking a hypothesis called the asymptotic factorization property [1]. This property has been shown to be satisfied for a class of driven systems having short-range spatial correlations such as the zero range process (ZRP).

The ZRP is one of the simplest example of driven interacting many-particle systems which do not satisfy detailed balance and have nonequilibrium steady states 12]. Previously, the ZRP has been mainly considered as model-system for various mass transport processes and has been used to study the phenomenon of condensation transition in nonequilibrium systems [13. Recently, it has been demonstrated 14 that the systems governed by the ZRP has a simple thermodynamic structure where a suitably defined intensive variable, like equilibrium chemical potential, indeed equalizes for two such systems in contact. There is also a corresponding fluctuationresponse relation between the compressibility and the fluctuation in the particle-number, which is satisfied exactly for a system in contact with a particle reservoir. 
The main advantage of studying these simple models like the ZRP was that the steady-state probability distributions, which have simple factorized forms, can be calculated exactly and therefore various features of driven systems in contact can be studied analytically. Due to the simple form of the steady-state distribution, it was also possible to analyze the role of dynamics at the contact between two systems.

However for driven systems with nontrivial steadystate properties, the situation is expected to be far more complex. Here we consider a simple model of a driven interacting many-particle system known as Katz-LebowitzSpohn (KLS) model [15, 16]. The KLS model, first introduced to study fast ionic conductors [17], is a paradigm in nonequilibrium statistical mechanics. The model describes a stochastic lattice gas of charged particles which is homogeneously driven by a constant externally applied electric field. Initially, the primary motivation behind studying the KLS model was to understand the nontrivial spatial structures and phase transitions in generic bulk-driven systems with nonequilibrium steady states. Since the introduction, the KLS model has been studied intensively. By now, the phase diagram in the plane of temperature and electric field strength is quite well known, mainly from extensive simulations [15, 16, 18, 19] as well as the results from mean-field theory [20] and renormalization-group analysis of a continuum version of the model 19, 21]. However there is still no well founded thermodynamic theory for these driven interacting manyparticle systems.

In this paper, we explore the "equilibration" between two driven lattice gases when they are brought into contact. Recently we studied the KLS model, with repulsive nearest-neighbor interactions among particles [22], which revealed a simple but approximate thermodynamic structure. Here we extend our previous studies to the systems with attractive interactions as well. Interestingly, both for attractive as well as for repulsive interactions and for a wide range of parameter values, we find that, to a very good approximation, there is an intensive thermodynamic variable, like equilibrium chemical potential, which determines the final steady state while two systems are allowed to exchange particles. Consequently, the zeroth law of thermodynamics as well as the fluctuation-response relation between the compressibility and the fluctuations in particle-number are satisfied remarkably well in a wide range of parameter values.

However there are also observable deviations from this simple thermodynamic structure, especially at high interaction strengths and large driving fields. We explain these deviations by expressing the asymptotic factorization property, which was initially proposed by Bertin et al. in [11, 14] and later discussed by us for driven lattice gases in 22], in a modified form where contributions to the large deviation functions due to the contact dynamics are identified. To illustrate the origin of these deviations,

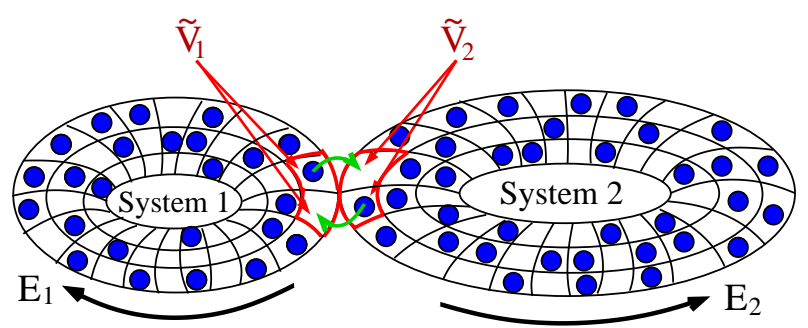

FIG. 1. A schematic diagram of two nonequilibrium steady states in contact with the contact region $\tilde{V}_{1}$ and $\tilde{V}_{2}$. Particles are allowed to be exchanged through the contact region $\tilde{V}_{1}$ and $\tilde{V}_{2}$. In this process the total number of particles $N=N_{1}+N_{2}$ are conserved where $N_{1}$ and $N_{2}$ are the number of particles in system 1 and system 2 respectively.

we study the nontrivial role of the contact dynamics using first a simple variant of the ZRP and later the KLS model. We find that, depending on the various parameter values, the contact dynamics can amount to an excess chemical potential across the contact for the variant of the ZRP as well as the KLS model. This excess chemical potential is generally a-priori unknown, and in some sense arbitrary for an arbitrarily chosen contact dynamics. Therefore, it may not be always possible to assign, to the individual systems, an intensive variable which is independent of the contact between the two systems, thus accounting for the deviations from the zeroth law.

Here is a brief outline of the paper. In section II we describe the model, in section III we present the numerical results concerning the zeroth law for the KLS model and possible deviations from the law. In section IV, we discuss the excess chemical potential for a variant of the ZRP as well as a variant of the equilibrium KLS model. In section $\mathrm{V}$, we describe how the large deviation principle can be written in a modified form which can capture the deviations from the zeroth law and then discuss the excess chemical potential for the KLS model. At the end, we summarize. In Appendix A, we give proof of the ansatz for the steady-state probability distribution in the case of the ZRP and, in Appendix B, we discuss the fluctuation-response relation for the ZRP as well as for the KLS model.

\section{MODEL}

We consider stochastic lattice gases of charged particles which are driven out of equilibrium by constant externally applied electric fields in the bulk and which therefore have spatially homogeneous steady states [15]. Particles move on a discrete lattice and jump stochastically from one site to any of its nearest-neighbor sites, preferably towards the direction of the external driving field of magnitude $E$. Due to hardcore repulsion among 
particles, a lattice site can be occupied by at most one particle. In addition, particles may also interact with each other through a nearest-neighbor pair-potential of interaction strength $K$. We define the occupation variable $\eta(\mathbf{r})$ at a site $\mathbf{r} \equiv\left\{r_{x}, r_{y}\right\}$ where $\eta(\mathbf{r})=0,1$ : if a site $\mathbf{r}$ is occupied $\eta(\mathbf{r})=1$ otherwise $\eta(\mathbf{r})=0$. We consider two systems of lattice gases where system 1 and system 2 are confined respectively in volume $V_{1}$ and volume $V_{2}$ (see Fig. 11. When two such systems are brought into contact, they are connected at a finite set of points $V_{1}^{\prime}$ and $V_{2}^{\prime}$, subsets of $V_{1}$ and $V_{2}$ respectively $\left(V_{1}^{\prime}, V_{2}^{\prime} \ll V_{1}, V_{2}\right)$ and, while in contact, they can exchange particles with each other. The energy function $H$ of the combined two systems is given by

$$
H=K_{1} \sum_{\left\langle\mathbf{r}_{1}, \mathbf{r}_{1}^{\prime}\right\rangle} \eta\left(\mathbf{r}_{1}\right) \eta\left(\mathbf{r}_{1}^{\prime}\right)+K_{2} \sum_{\left\langle\mathbf{r}_{2}, \mathbf{r}_{2}^{\prime}\right\rangle} \eta\left(\mathbf{r}_{2}\right) \eta\left(\mathbf{r}_{2}^{\prime}\right)
$$

where $\langle *, *\rangle$ denotes sum over nearest-neighbor sites with $\mathbf{r}_{1}, \mathbf{r}_{1}{ }^{\prime} \in V_{1}$ and $\mathbf{r}_{2}, \mathbf{r}_{\mathbf{2}}{ }^{\prime} \in V_{2}, K_{1}$ and $K_{2}$ strength of interactions among particles for the respective systems. Note that systems may in general have different microscopic dynamics depending on the interaction strengths $K_{1}$ and $K_{2}$. Constant driving fields, $E_{1}$ and $E_{2}$ respectively in systems 1 and 2, are applied along $x$-directions, with periodic boundary conditions imposed in both $x$ and $y$ directions. Driven bilayer systems have been considered before where particles can jump from one layer to the other at any site [23]. However here we consider the case where particles can jump from one system to the other through a very small contact region between the systems.

We choose jump rates of particles such that they satisfy the local detailed balance condition [15]. A pair of nearest -neighbor sites, located at $\mathbf{r}$ and $\mathbf{r}^{\prime}$, in a configuration $C$ are chosen randomly and an attempt is made to interchange the occupation variables where the attempted final configuration is denoted by $C^{\prime}$. Let us denote the corresponding transition rate from configuration $C$ to $C^{\prime}$ as $w\left(C^{\prime} \mid C\right)$. For movements of particles inside the same system (i.e., particles not jumping from one system to the other), a quantity $\Delta(E)=H\left(C^{\prime}\right)-H(C)-E\left(r_{x}-r_{x}^{\prime}\right)$, which depends on the driving field $E$, is defined where $r_{x}$ denotes the $x$-component of the position vector $\mathbf{r}$ and $H(C)$ is the energy of the configuration $C$. The transition rate is assigned to be

$$
w\left(C^{\prime} \mid C\right)=\min \left\{1, e^{-\beta \Delta(E)}\right\}
$$

where $\beta$ is the inverse temperature of the heat bath. When the chosen pair of sites are such that a jump is attempted from one system to the other across the contact, the transition rate is assigned to be

$$
w\left(C^{\prime} \mid C\right)=\min \left\{1, e^{-\beta \Delta(0)}\right\}
$$

where $\Delta(0)=H\left(C^{\prime}\right)-H(C)$. Note that there is no field along the bonds connecting the two systems. In the simulations, we consider two-dimensional systems $(V=$
$L \times L)$ with periodic boundaries in both directions. We put $\beta=1$ throughout the paper.

When $E_{1}=E_{2}=0$, the jump rates satisfy the detailed balance condition and the configuration $C$ of the combined system has the Boltzmann probability distribution $P(C) \sim \exp [-H(C)]$. For $E_{1}, E_{2} \neq 0$, there is a constant current in the steady state. However, unlike in equilibrium, the nonequilibrium steady-state probability distribution in general is not given by the Boltzmann distribution with an a-priori known energy function and, except for a few cases (e.g., with only hard-core interactions), the steady-state probability distribution is not known. When two systems are brought into contact, they can exchange particles with the total number of particles $N=N_{1}+N_{2}$ conserved where $N_{1}=\sum_{\mathbf{r}_{1} \in V_{1}} \eta\left(\mathbf{r}_{1}\right)$ and $N_{2}=\sum_{\mathbf{r}_{2} \in V_{2}} \eta\left(\mathbf{r}_{\mathbf{2}}\right)$ are number of particles in system 1 and system 2, respectively. In the sections below, we consider the cases where the conserved quantity is the number of particles and therefore attempt to define an intensive variable, called chemical potential in analogy with equilibrium, which is conjugate to the conserved particle-number.

\section{NUMERICAL RESULTS}

\section{A. The Zeroth Law}

First let us describe the zeroth law in the context of equilibrium systems. We consider three systems and perform the following three thought-experiments which are schematically presented in Fig. 2, In the first experiment, system 1 and system 3 are brought into contact and allowed to exchange particles with each other. System 1 and system 3 eventually equilibrate and reach a final equilibrium state with constant average densities $n_{1}$ and $n_{3}$ respectively. In the second experiment, system 2 and system 3 are separately brought into contact and allowed to exchange particles. In this case, the initial density of system 2 is tuned such that system 3 has the same final density as that of system 3 in the first experiment. Let us denote the final equilibrium densities for system 2 and 3 in the second experiment as $n_{2}$ and $n_{3}$ respectively. Now in the third experiment, system 1 and system 2 with initial densities $n_{1}$ and $n_{2}$ respectively are brought into contact and allowed to exchange particles. One could ask what the final densities in this case would be. The zeroth law of thermodynamics provides the answer that the final densities will not change any more and will be exactly equal to the respective initial densities. Thus the zeroth law allows us to assign to an equilibrium system an intensive thermodynamic variable called the chemical potential which equalizes for two systems in contact. Note that, in Fig. 2 if one compares the density profiles of two systems being in the same column, the corresponding average density profiles would be 
exactly same.

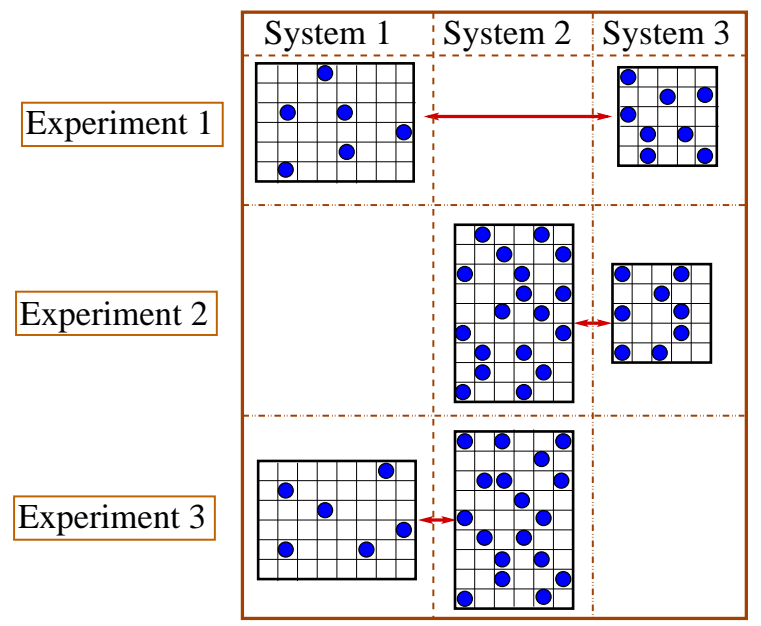

FIG. 2. Schematic diagram to test the zeroth law of thermodynamics for equilibrium systems using a sequence of three thought-experiments where three systems (for simplicity, shown in two dimensions) are separately kept in contact with each other and allowed to equilibrate. Experiment 1: System 1 and system 3 in contact. Experiment 2: System 2 and system 3 in contact. Experiment 3: System 1 and system 2 in contact.

We use the above strategy to test the zeroth law for systems in nonequilibrium steady states. We perform the same set of numerical experiments as described above, but now with two of the systems driven out of equilibrium due to external driving fields present in the bulk of the individual systems. Interestingly, similar to the equilibrium case, we observe that, for various values of interaction strengths, driving fields and densities, the zeroth law is quite well satisfied, i.e, if two driven lattice gases are separately "equilibrated" with a common system with a fixed density, they will also equilibrate amongst themselves. Two such examples are given below.

1. Example for driven systems with attractive interactions - In Fig. 3 we consider three systems, a driven system 1 with $K=-1, E=6$, a driven system 2 with $K=-0.75, E=4$ and an equilibrium system 3 with $K=0, E=0$. First, system 1 with density $n_{1} \simeq 0.60$ and system 2 with density $n_{2} \simeq 0.29$ are separately "equilibrated" with system 3 with a fixed density $n_{3} \simeq 0.15$. We then find that system 1 and system 2 , with the initial densities $n_{1} \simeq 0.60$ and $n_{2} \simeq 0.29$ respectively, "equilibrate" with each other such that, to a very good approximation, the respective final steady-state values of densities $n_{1}^{\prime} \simeq 0.60$ and $n_{2}^{\prime} \simeq 0.29$ remain almost unchanged.

2. Example for driven systems with repulsive in-

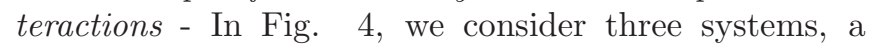
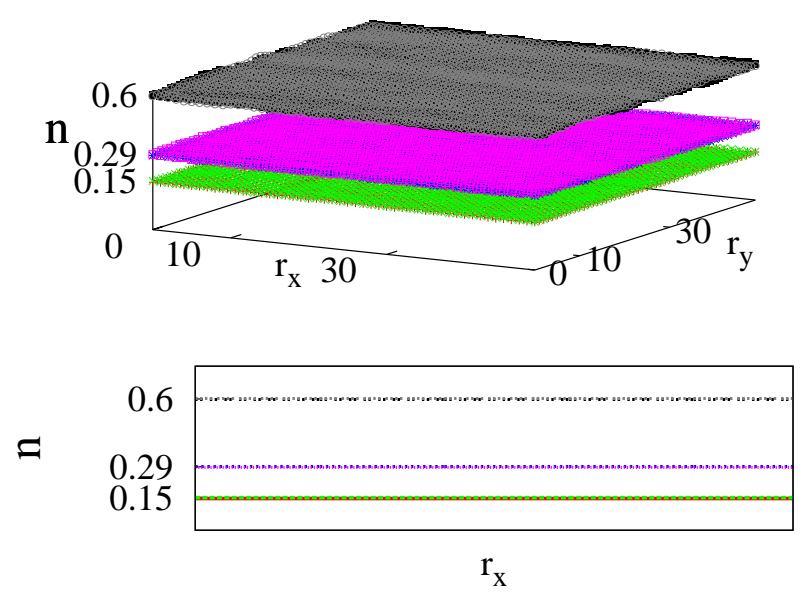

FIG. 3. Numerical experiments to test the zeroth law. Top panel: Average densities as a function of position co-ordinates $r_{x}$ and $r_{y}$. Bottom panel: Cross-sections (along $x$-direction) of the density profiles. Driven system 1: $K=-1, E=6$ (top density profiles). Driven system 2: $K=-0.75, E=4$ (middle density profiles). Equilibrium system $3: K=0, E=0$ (bottom density profiles). All systems considered here are with same volume $V=50 \times 50$. Experiment 1 : System 1 with density $n_{1} \simeq 0.60$ (top grey profile) "equilibrated" with system 3 with density $n_{3} \simeq 0.15$ (bottom red profile). Experiment 2: System 2 with density $n_{2} \simeq 0.29$ (middle magenta profile) "equilibrated" with system 3 with density $n_{3} \simeq 0.15$ (bottom green profile). Experiment 3: System 1 with density $n_{1}^{\prime}$ (top black profile) "equilibrated" with system 2 with density $n_{2}^{\prime}$ (middle blue profile) where $n_{1}^{\prime} \simeq n_{1}$ and $n_{2}^{\prime} \simeq n_{2}$.

driven system 1 with $K=3.75, E=6$, a driven system 2 with $K=1.5, E=5$ and an equilibrium system 3 with $K=0.75, E=0$. System 1 with density $n_{1} \simeq 0.40$ and system 2 with density $n_{2} \simeq 0.57$ are separately "equilibrated" with system 3 with a fixed density $n_{3} \simeq 0.76$. In Fig. 4 one could see that system 1 and system 2 with the above initial densities $n_{1} \simeq 0.40$ and $n_{2} \simeq 0.57$ respectively equilibrate with each other with the respective final densities $n_{1}^{\prime} \simeq 0.39$ and $n_{2}^{\prime} \simeq 0.58$. Also in this case, the zeroth law is satisfied to a good approximation where final densities $n_{1}^{\prime}$ and $n_{2}^{\prime}$ remain almost same as the initial densities.

Interestingly, as seen in Figs. 3 and 4 two systems in contact have homogeneous density profiles even when one or both of them may be driven. The driven systems are indeed far away from equilibrium since the numerical values of currents in the nonequilibrium steady states considered in Figs. 3 and 4 are near to the respective maximum values of currents (data not shown). Moreover in the top panel of Fig. 4 system 1 with density $n_{1} \simeq 0.40$ has a homogeneous disordered state in contrast to an ordered state for the corresponding equilibrium system. The equilibrium system, with the same interaction strength $K=3.75$ and the same density, has 

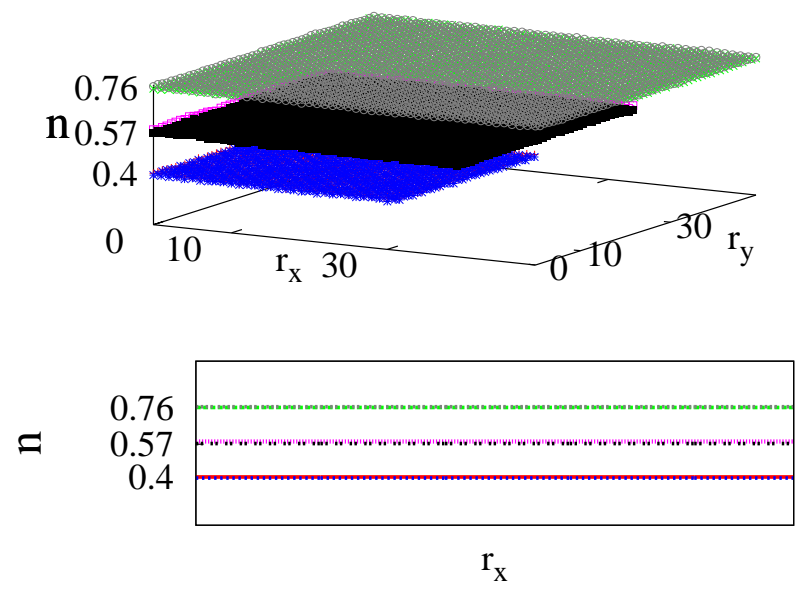

FIG. 4. Numerical experiments to test the zeroth law. Top panel: Average densities as a function of position co-ordinates $r_{x}$ and $r_{y}$. Bottom panel: Cross-sections (along $x$-direction) of the density profiles. Driven system 1: $K=3.75, E=6$ and $V=32 \times 32$ (bottom density profiles). Driven system 2 : $K=1.5, E=5$ and $V=40 \times 40$ (middle density profiles). Equilibrium system 3: $K=0.75, E=0$ and $V=50 \times 50$ (top density profiles). Experiment 1: System 1 with density $n_{1} \simeq$ 0.40 (bottom red profile) "equilibrated" with system 3 with density $n_{3} \simeq 0.76$ (top grey profile). Experiment 2: System 2 with density $n_{2} \simeq 0.57$ (middle black profile) "equilibrated" with system 3 with density $n_{3} \simeq 0.76$ (top green profile). Experiment 3: System 1 with density $n_{1}^{\prime}$ (bottom blue profile) "equilibrated" with system 2 with density $n_{2}^{\prime}$ (middle magenta profile) where $n_{1}^{\prime} \simeq n_{1}$ and $n_{2}^{\prime} \simeq n_{2}$.

a symmetry-broken ordered phase with a checkerboardlike pattern where sub-lattice densities are different [16].

Importantly, the macroscopic properties like densities do indeed depend on the driving field when a driven system 1 is kept in contact with an equilibrium system 2 with a fixed density $n_{2}$. This can be seen in the behavior of density $n_{1}$ of a driven system as a function of driving field $E_{1}$. We consider the driven systems which were previously used to test the zeroth law in Figs. 3and 4 . In the top panel of Fig. 15, densities of two driven systems with attractive interactions, with $K_{1}=-1$ and $K_{1}=-0.75$, have been plotted as a function of the driving fields $E_{1}$ in the respective systems where both the systems are separately kept in contact with an equilibrium system with $K_{2}=0$ and a fixed density $n_{2} \simeq 0.15$. In the bottom panel of Fig. 5, densities of two other driven systems with repulsive interactions, with $K_{1}=3.75$ and $K_{1}=1.5$, have been plotted as a function of the driving fields $E_{1}$ in the respective systems where both the systems are now separately kept in contact with an equilibrium system with $K_{2}=0.75$ and a fixed density $n_{2} \simeq 0.76$. One could see that the densities vary quite significantly, almost by an amount of $10 \%$ or more from the respective equilibrium values depending on the interaction strengths, when
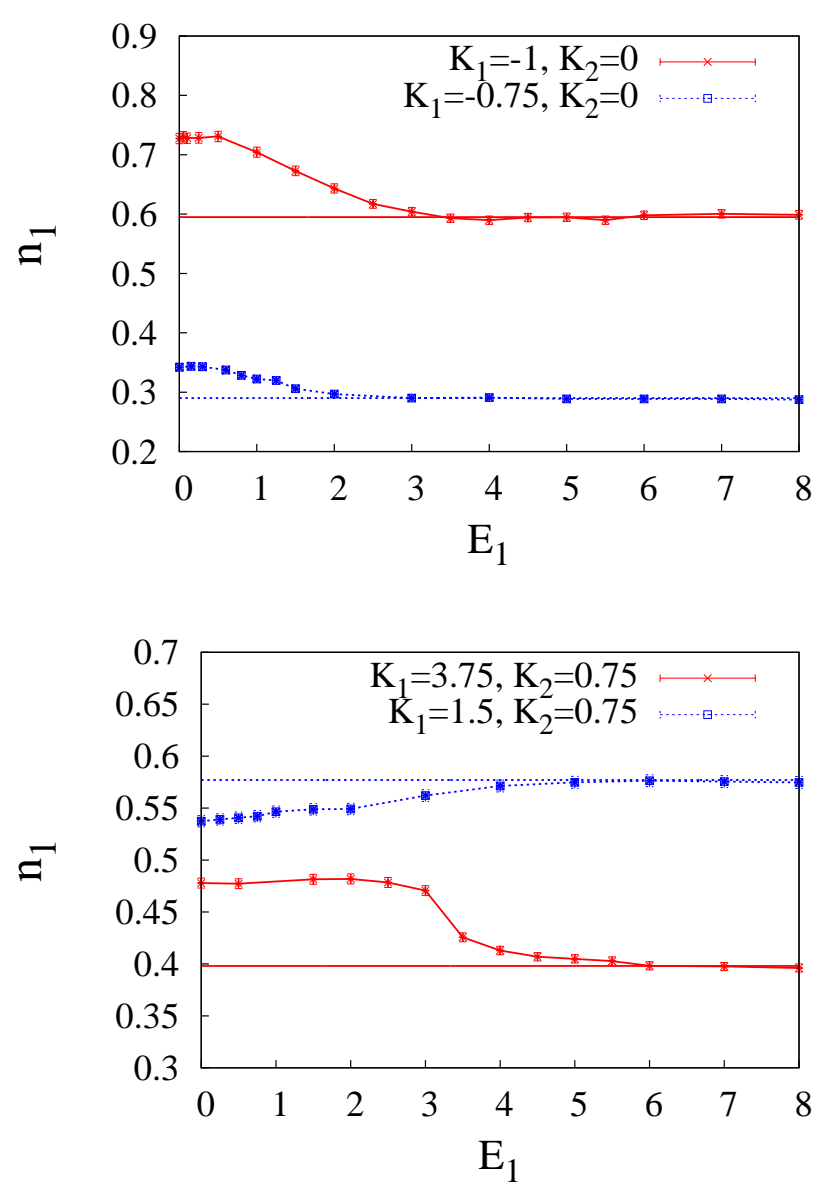

FIG. 5. Density $n_{1}$ of driven system 1 as a function of driving field $E_{1}$ when the system 1 is in contact with an equilibrium system 2 with a fixed density $n_{2}$. Top panel: Densities of two driven systems with interaction strengths $K_{1}=-1$ and $K_{1}=-0.75$ plotted as a function of respective driving fields $E_{1}$ when the systems are separately in contact with an equilibrium system with $K_{2}=0$ and $n_{2} \simeq 0.15$. Bottom panel: Densities of two driven systems with interaction strengths $K_{1}=3.75$ and $K_{1}=1.5$ plotted as a function of respective driving fields $E_{1}$ when the systems are separately in contact with an equilibrium system with $K_{2}=0.75$ and $n_{2} \simeq 0.76$.

the driving field varies from $E_{1}=0$ to a large value $E_{1} \gg K_{1}$.

Provided that the zeroth law is satisfied, one can define a chemical potential even for a driven system as following. A driven system is kept in contact with an equilibrium one and allowed to reach a steady state. Then, in the steady state, one can simply assign the chemical potential of the equilibrium system to the driven one. For nonzero interaction strengths $K \neq 0$, even the equilibrium chemical potential $\mu$ cannot be calculated directly as one does not know the explicit form of $\mu(n)$ as a function of density $n$. However, for equilibrium system with 


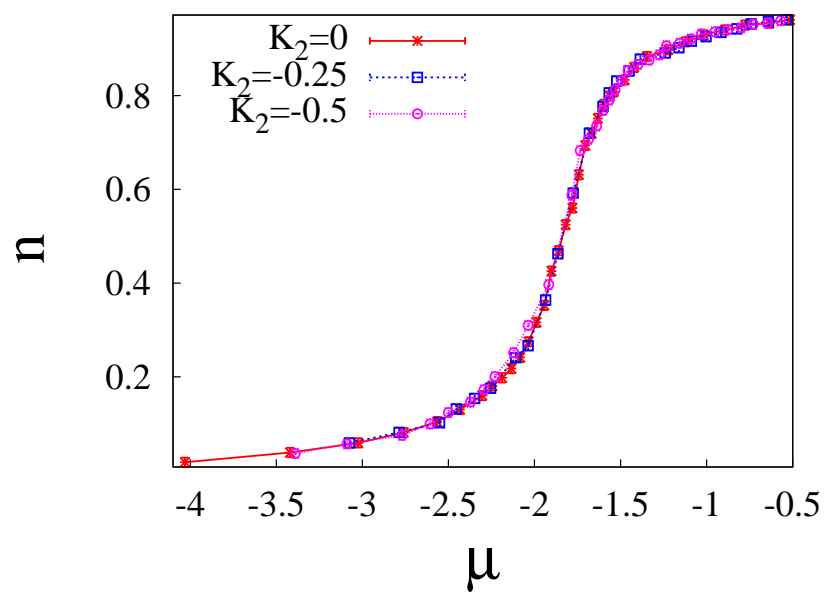

FIG. 6. Density $n$ vs. chemical potential $\mu$ is plotted for a driven system with $K_{1}=-1, E_{1}=6$ which is separately kept in contact with three equilibrium systems with interaction strengths $K_{2}=0, K_{2}=-0.25$, and $K_{2}=-0.5$. The collapse of the curves agrees well with the zeroth law.

noninteracting hardcore particles $K=0$, the chemical potential $\mu$ can be expressed as a function of density $n$ where

$$
\mu=\ln \left(\frac{n}{1-n}\right),
$$

by using the relation $\mu=-(\partial s / \partial n)$ where $s=-[n \ln n+$ $(1-n) \ln (1-n)]$ is the equilibrium entropy per lattice site. Since the zeroth law is exactly satisfied in equilibrium, the chemical potential for any equilibrium system with $K \neq 0$ can be measured by keeping the system in contact with an equilibrium system with $K=0$ and then assigning a chemical potential for a system with $K=0$ to that with $K \neq 0$. In Fig. [6 we plot the density $n$ as a function of chemical potential $\mu$ when a driven system with $K_{1}=-1, E_{1}=6$ is separately kept in contact with three equilibrium systems with various interaction strengths $K_{2}=0, K_{2}=-0.25$, and $K_{2}=-0.5$. Given that the zeroth law is satisfied, the various curves for density as a function of chemical potential should fall on each other. The collapse of curves in Fig. 6 indeed agrees quite well with the zeroth law.

We have studied the dependence of densities on the system sizes as well. We find that the densities of two systems in contact with each other, when one or both systems may be driven, are almost independent of system sizes. In Fig. 7 we plot the density $n$ as a function of chemical potential $\mu$ for various system sizes, and for various values of interaction strengths and driving fields. The densities seem to only depend on the interaction strengths and driving fields of the respective systems.

In Figs. 3 and 4 we have tested the zeroth law only for a particular set of values of densities. We now study

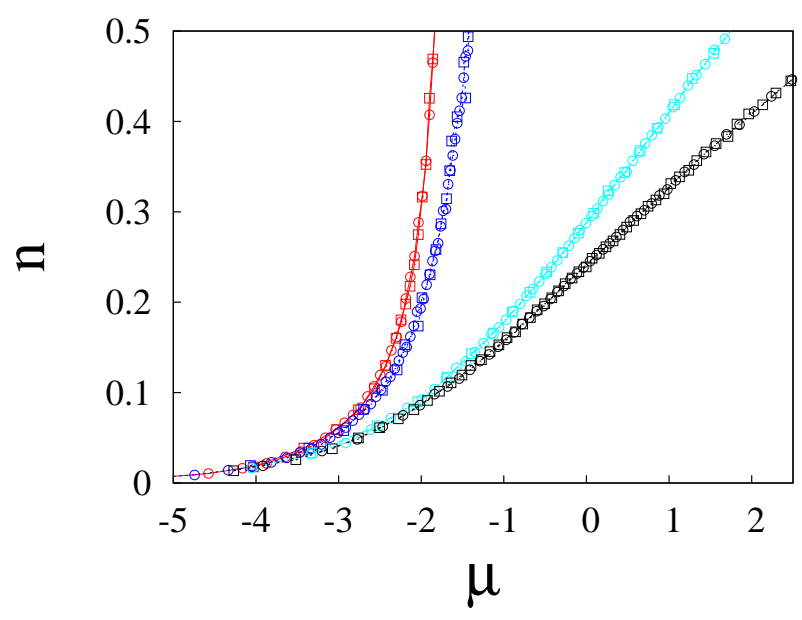

FIG. 7. Density $n$ as a function of chemical potential $\mu$ for driven systems (fixed $K_{1}, E_{1}$ ) with different values of system size $V_{1}$ in contact with an equilibrium system of noninteracting hardcore particles $\left(K_{2}=0, E_{2}=0\right)$ with different values of system size $V_{2}$. Circles correspond to the case when the system 1 with $V_{1}=32 \times 32$ is in contact with system 2 with $V_{2}=32 \times 32$. Squares correspond to the case when the system 1 with $V_{1}=20 \times 20$ is in contact with system 2 with $V_{2}=100 \times 100$. For the curves from left to right: (1) two curves (red) for systems with $K_{1}=-1, E_{1}=6$, (2) two curves (blue) for systems with $K_{1}=-0.75, E_{1}=4$, (3) two curves (sky blue) for systems with $K_{1}=1, E_{1}=6$, and (4) two curves (black) for systems with $K_{1}=2, E_{1}=2$.

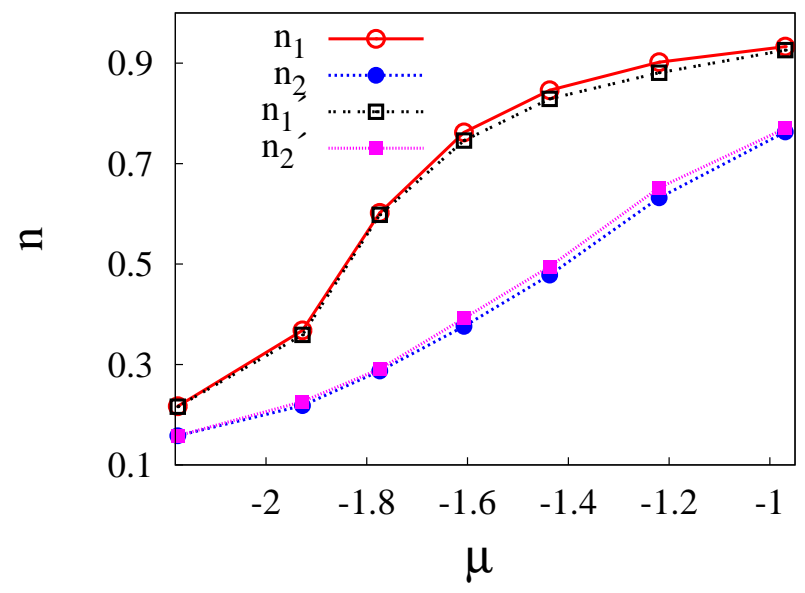

FIG. 8. Final densities $n_{1}^{\prime}$ and $n_{2}^{\prime}$ for two driven systems, respectively with $K=-1, E=6$ and $K=-0.75, E=4$, being in contact with each other are compared with their respective initial densities $n_{1}$ and $n_{2}$. The initial densities correspond to those obtained by keeping the driven systems separately in contact with an equilibrium system of a fixed chemical potential $\mu$. If the zeroth law is satisfied, the initial and final densities should be exactly the same for a given $\mu$.

the zeroth law for various other densities where the in- 


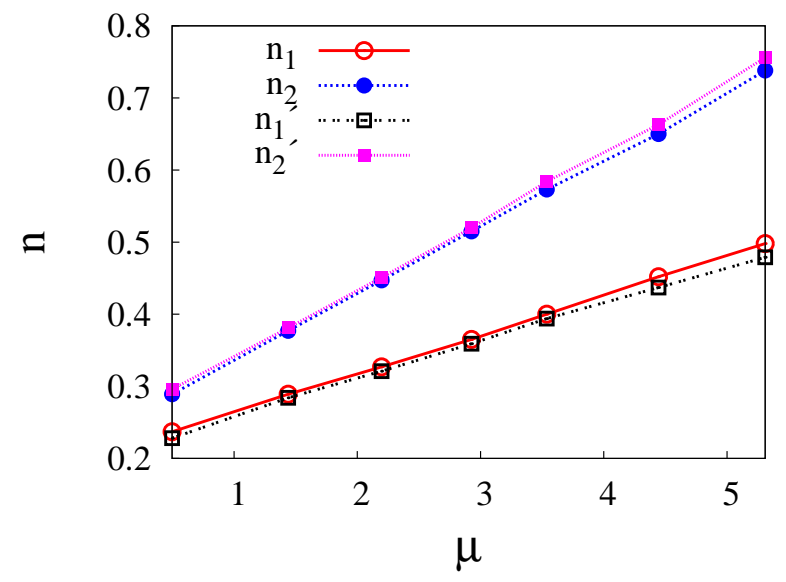

FIG. 9. Final densities $n_{1}^{\prime}$ and $n_{2}^{\prime}$ for two driven systems, respectively with $K=3.75, E=6$ and $K=1.5, E=5$, being in contact with each other are compared with their respective initial densities $n_{1}$ and $n_{2}$. The initial densities correspond to those obtained by keeping the driven systems separately in contact with an equilibrium system of a fixed chemical potential $\mu$. If the zeroth law is satisfied, the initial and final densities should be exactly the same for a given $\mu$.

teraction strengths and driving fields are kept fixed. We consider driven systems 1 and 2 with respective densities $n_{1}$ and $n_{2}$ where the systems are separately equilibrated with an equilibrium system 3 with density $n_{3}$ and corresponding chemical potential $\mu$. Then system 1 and system 2 with the respective initial densities $n_{1}$ and $n_{2}$ are equilibrated with each other where they eventually reach final densities $n_{1}^{\prime}$ and $n_{2}^{\prime}$. Clearly, if the zeroth law is satisfied, then the corresponding initial and final densities would be exactly the same, i.e., $n_{1}=n_{1}^{\prime}$ and $n_{2}=n_{2}^{\prime}$. We consider the systems as previously discussed in Figs. 3 and 4. In Fig. 8, we plot $n_{1}$ and $n_{1}^{\prime}$ for system 1 with $K=-1, E=6$ and $n_{2}$ and $n_{2}^{\prime}$ for system 2 with $K=-0.75, E=4$ as a function of chemical potential $\mu$ of the equilibrium system 3 , with $K=E=0$. Similarly, in Fig. 9] we plot $n_{1}$ and $n_{1}^{\prime}$ for system 1 with $K=3.75$, $E=6$ and $n_{2}$ and $n_{2}^{\prime}$ for system 2 with $K=1.5, E=5$ as a function of chemical potential $\mu$ of the system 3 , with $K=0.75, E=0$. Although, the zeroth law is satisfied to a good approximation, there are indeed small but observable deviations from the law, i.e., up to $5 \%$ deviations in the final densities from the corresponding initial density values. In the next section we discuss the deviations from the zeroth law in more detail.

\section{B. Deviations from the Zeroth Law}

In the previous section, we have seen that, for a large parameter range, the driven lattice gases have an approximate but remarkably simple thermodynamic structure.
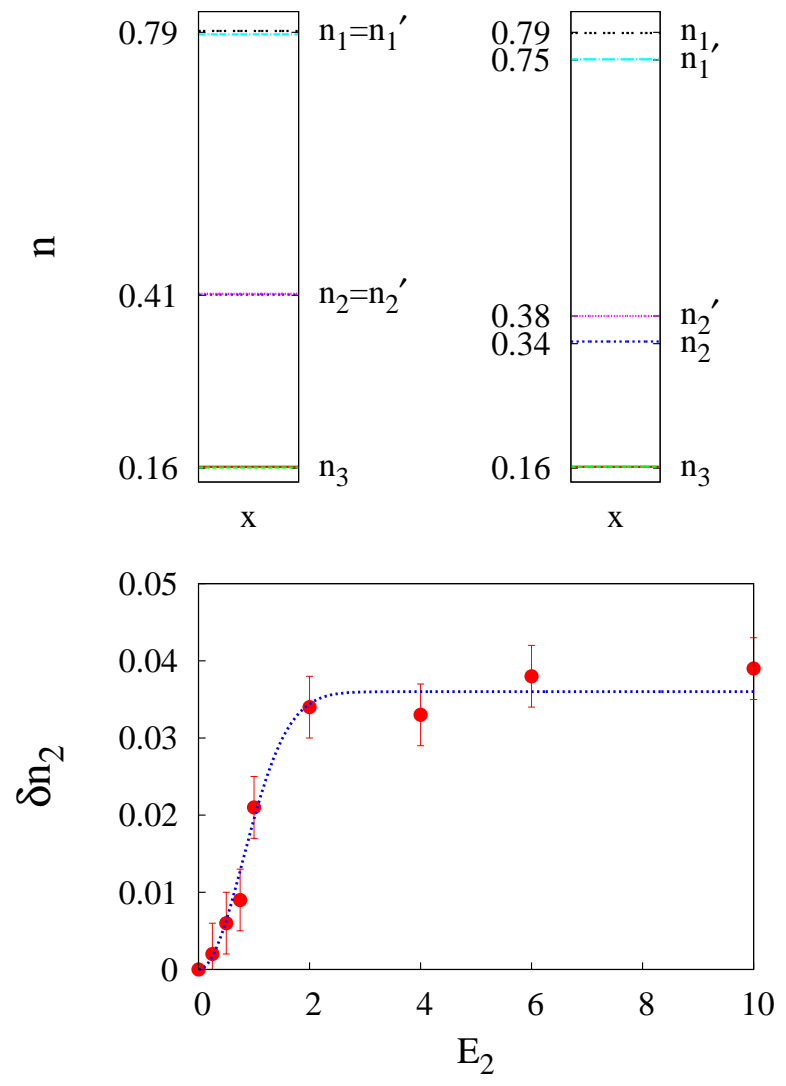

FIG. 10. In top panel, the cross-sections (along $x$-direction) of the density profiles are plotted. Systems 1, 2 and 3 (all with same size $V=50 \times 50$ ) have interaction strengths $K=-1$ (top density profiles), $K=-0.75$ (middle density profiles) and $K=0$ (bottom density profiles) respectively. First, system 1 with density $n_{1}$ and system 2 with density $n_{2}$ are separately equilibrated with system 3 with density $n_{3}$. Then system 1 and 2, with initial densities $n_{1}$ and $n_{2}$ respectively, are equilibrated with each other where the final steady-state densities are $n_{1}^{\prime}$ and $n_{2}^{\prime}$ respectively. Top panel (left): For equilibrium systems where $n_{1}=n_{1}^{\prime}$ and $n_{2}=n_{2}^{\prime}$. Top panel (right): For the case when system 2 driven with field $E_{2}=10$ with $n_{1} \neq n_{1}^{\prime}$ and $n_{2} \neq n_{2}^{\prime}$. Bottom panel: Difference in density $\delta n_{2}=n_{2}^{\prime}-n_{2}$ is plotted versus driving field $E_{2}$ for system 2 . The blue line, which is a fitting function $\left(a-b e^{-\kappa E_{2}^{2}}\right)$ with $a=0.036, b=0.036$ and $\kappa=0.78$, is a guide to the eye.

However, it should be noted that there are also larger observable deviations from the simple thermodynamic law as discussed next.

In Fig. 10, we perform numerical experiments similar to that discussed before (e.g., see Figs. 3 and 4) where system 1 with density $n_{1}$ and system 2 with density $n_{2}$ are separately equilibrated with system 3 with a fixed density $n_{3}$. Then system 1 and system 2 , with the respective initial densities $n_{1}$ and $n_{2}$, are equilibrated with each other where the final steady-state densities are $n_{1}^{\prime}$ 
and $n_{2}^{\prime}$ respectively. In the top-left panel of Fig. 10 , six density profiles along $x$-direction are plotted for equilibrium systems 1, 2, and 3. Expectedly, for equilibrium systems, the zeroth law is exactly satisfied where $n_{1}=n_{1}^{\prime} \simeq 0.79$ (top density profiles) and $n_{2}=n_{2}^{\prime} \simeq 0.41$ (middle density profiles), i.e., initial densities are equal to the respective final densities. In the top-right panel of Fig. 10, six density profiles along $x$-direction are plotted when systems 1 and 3 are equilibrium systems but system 2 is driven with a large field $E_{2}=10$. In this case, the zeroth law is observed to be violated significantly where $n_{1} \simeq 0.79 \neq n_{1}^{\prime} \simeq 0.75$ (top density profiles) and $n_{2} \simeq 0.34 \neq n_{2}^{\prime} \simeq 0.38$ (middle density profiles), i.e., final densities change appreciably as compared to the respective initial densities. In the bottom panel of Fig. 10, the density difference $\delta n_{2}=n_{2}^{\prime}-n_{2}$ is plotted as a function of the driving field $E_{2}$ in system 2 .

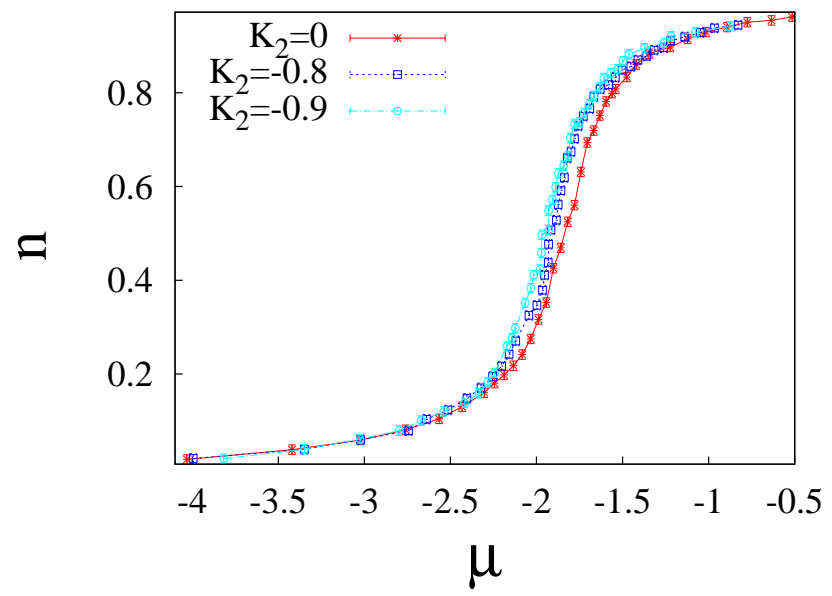

FIG. 11. Density $n$ as a function of chemical potential $\mu$ for a driven system with $K_{1}=-1, E_{1}=6$ which is separately kept in contact with three equilibrium systems with interaction strengths $K_{2}=0, K_{2}=-0.8$, and $K_{2}=-0.9$.

One can also observe the deviations from the zeroth law which is now studied in a slightly different way as follows. We first try to assign a chemical potential to a driven system by keeping the system separately in contact with various equilibrium systems and then compare density vs. chemical potential curves. In Fig. 11, we plot density $n$ as a function of chemical potential $\mu$ for a driven system, with $K_{1}=-1, E_{1}=6$, which is separately kept in contact with three equilibrium systems with the following interaction strengths: $K_{2}=0$, $K_{2}=-0.8$ and $K_{2}=-0.9$. If the zeroth law was satisfied, all the curves should fall on each other. However, we observe that there are significant deviations which occur especially around density $n=1 / 2$. In Fig. 12, we plot $n$ vs. $\mu$ for another driven system, with repulsive interaction $K_{1}=3.75, E_{1}=6$, being separately in contact with equilibrium systems with $K_{2}=0.75$ and $K_{2}=1$.

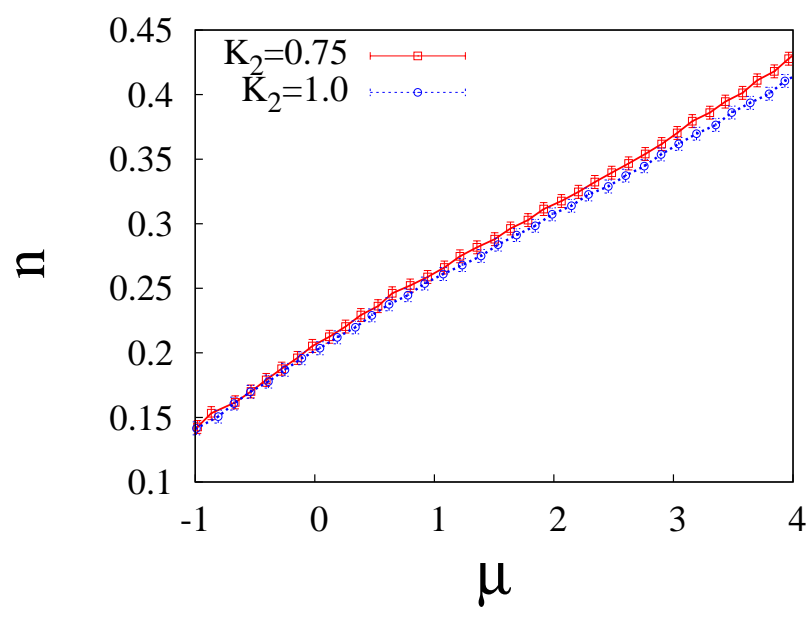

FIG. 12. Density $n$ as a function of chemical potential $\mu$ for a driven system with $K_{1}=3.75, E_{1}=6$ which is separately kept in contact with two equilibrium systems with interaction strengths $K_{2}=0.75$ and $K_{2}=1$.

We again see deviations from the zeroth law when the density approaches $n=1 / 2$.

\section{ROLE OF CONTACT DYNAMICS: EXACT RESULTS}

\section{A. Excess Chemical Potential in the ZRP}

We now study a simple class of driven systems, known as the zero range process (ZRP) [12], to understand the role of contact dynamics in the context of equalization of thermodynamic variables for driven systems. The ZRP is defined on a discrete lattice where, unlike the KLS model, there is no restriction on the occupation number of a site, i.e., the lattice sites can be occupied by more than one particle. The jump rate of a particle out of any site is assumed to depend on the number of particles on the site.

For simplicity, we consider two one-dimensional rings, ring 1 and ring 2 , consisting of $L_{1}$ and $L_{2}$ sites respectively. The rings are kept in contact with each other so that they can exchange particles through the contact area. In the $\alpha$ th ring $(\alpha=1,2)$, any site $i_{\alpha}$ $\left(i_{\alpha}=1,2, \ldots, L_{\alpha}\right)$ is occupied with $n_{i_{\alpha}}$ particles. Any configuration $C$ can be specified, by using the occupation numbers at all sites of two rings, $C \equiv\left(\left\{n_{i_{1}}\right\},\left\{n_{i_{2}}\right\}\right)$. Two rings are connected at two sites, say $i_{1}=1$ and $i_{2}=1$, through which particles can be exchanged between the rings. The dynamics is defined as follows: a particle at site $i_{\alpha}$ can jump only in one direction, say to the nearest neighbor site $i_{\alpha}+1$ in the clockwise direction (therefore 
violating detailed balance), with rate

$$
u_{\alpha}\left(n_{i_{\alpha}}\right)=v_{\alpha} \frac{f_{\alpha}\left(n_{i_{\alpha}}-1\right)}{f_{\alpha}\left(n_{i_{\alpha}}\right)} \text { with } \alpha=1,2
$$

where $f_{\alpha}\left(n_{i_{\alpha}}\right)$ is a function of occupation number $n_{i_{\alpha}}$ and the factor $v_{\alpha}$ is independent of $n_{i_{\alpha}}$. The form of the jump rates is the same as given in Eq. 5 irrespective of jumps in the bulk or jumps from one ring to the other. But the factors $v_{\alpha}$ can in general be different, i.e., $v_{\alpha}=v_{\alpha}^{(b)}$ when a particle jumps in the bulk of the $\alpha$ th ring and $v_{\alpha}=v_{\alpha}^{(c)}$ when a particle jumps at the contact from $\alpha$ th ring to the other ring. For the zero range process, the properties of nonequilibrium steady states while two systems are in contact has been previously studied, but only for a special case when $v_{\alpha}^{(b)}=v_{\alpha}^{(c)}$ and $v_{1}^{(c)}=v_{2}^{(c)}$ [14]. Here we consider the more general case when $v_{\alpha}^{(b)} \neq v_{\alpha}^{(c)}$ as well as $v_{1}^{(c)} \neq v_{2}^{(c)}$, i.e., the factor $v_{\alpha}$ taking different values at the bulk and the contact of the two rings. However, it would be easy to see that the factor $v_{\alpha}^{(b)}$ taking different values in two different rings does not change the steadystate properties. Therefore, now onwards, we put $v_{1}^{(b)}=$ $v_{2}^{(b)}=1$. Also, for notational simplicity, we denote $v_{1} \equiv$ $v_{1}^{(c)}$ and $v_{2} \equiv v_{2}^{(c)}$. For completeness, let us first discuss the special case when $v_{1}=v_{2}$ [14]. The steady state probability distribution can be written in a factorized form

$$
\begin{array}{r}
P\left(\left\{n_{i_{1}}\right\},\left\{n_{i_{2}}\right\}\right)=\frac{1}{Z_{N}}\left[\prod_{i_{1}=1}^{L_{1}} f_{1}\left(n_{i_{1}}\right) \prod_{i_{2}=1}^{L_{2}} f_{2}\left(n_{i_{2}}\right)\right] \\
\times \delta\left(N_{1}+N_{2}-N\right),
\end{array}
$$

where $N_{1}=\sum_{i_{1}=1}^{L_{1}} n_{i_{1}}$ and $N_{2}=\sum_{i_{2}=1}^{L_{2}} n_{i_{2}}$ are the number of particles in ring 1 and ring 2 respectively, $Z_{N}$ is the normalization constant. The delta function in the above equation takes into account that the total number of particles $N=N_{1}+N_{2}$ is conserved. Clearly in this case, the joint probability distribution $P\left(N_{1}, N_{2}\right)$ of particle numbers $N_{1}$ and $N_{2}$ can be written in a product form

$$
P\left(N_{1}, N_{2}\right)=\frac{Z_{1}\left(N_{1}\right) Z_{2}\left(N_{2}\right)}{Z_{N}}
$$

where $Z_{\alpha}\left(N_{\alpha}\right)=\sum_{\left\{n_{i_{\alpha}}\right\}} \prod_{i_{\alpha}=1}^{L_{\alpha}} f_{\alpha}\left(n_{i_{\alpha}}\right) \delta\left(\sum_{i_{\alpha}=1}^{L_{\alpha}} n_{i_{\alpha}}-\right.$ $N_{\alpha}$ ). As discussed in [11, 14], in this case, one can define an intensive variable $\mu_{\alpha}=-\partial \ln Z_{\alpha} / \partial N_{\alpha}$ which equalizes when two rings are kept in contact, i.e., $\mu_{1}=\mu_{2}$.

Now we discuss the general case when $v_{1} \neq v_{2}$ which leads to our main points. Interestingly, as shown in Appendix A, the steady state probability distribution can still be written in a factorized form

$$
\begin{aligned}
P\left(\left\{n_{i_{1}}\right\},\left\{n_{i_{2}}\right\}\right)= & \frac{1}{Z_{N}}\left[\prod_{i_{1}=1}^{L_{1}} f_{1}\left(n_{i_{1}}\right) \prod_{i_{2}=1}^{L_{2}} f_{2}\left(n_{i_{2}}\right)\right] \\
& \times e^{\tilde{\mu}_{1} N_{1}} e^{\tilde{\mu}_{2} N_{2}} \delta\left(N_{1}+N_{2}-N\right)
\end{aligned}
$$

where $\tilde{\mu}_{1}=\ln \left(1 / v_{1}\right)$ and $\tilde{\mu}_{2}=\ln \left(1 / v_{2}\right)$, which we call excess chemical potentials. Now the joint probability distribution $P\left(N_{1}, N_{2}\right)$ of particle-numbers $N_{1}$ and $N_{2}$ can be expressed as

$$
P\left(N_{1}, N_{2}\right)=\frac{Z_{1}\left(N_{1}\right) Z_{2}\left(N_{2}\right)}{Z_{N}} e^{\tilde{\mu}_{1} N_{1}} e^{\tilde{\mu}_{2} N_{2}} .
$$

The macrostate is obtained by maximizing $\ln P\left(N_{1}, N_{2}\right)$, i.e., $\partial \ln P\left(N_{1}, N_{2}\right) / \partial N_{1}=0$. Therefore it straightforwardly follows that

$$
\left(\frac{\partial \ln Z_{1}}{\partial N_{1}}+\tilde{\mu}_{1}\right)=\left(\frac{\partial \ln Z_{2}}{\partial N_{2}}+\tilde{\mu}_{2}\right)
$$

or, in other words, there indeed exists new intensive variable $\mu_{\alpha}^{\prime}=\left(-\partial \ln Z_{\alpha} / \partial N_{\alpha}+\ln v_{\alpha}\right)$, with $\alpha=1,2$, which takes the same values for two rings in contact, i.e., $\mu_{1}^{\prime}=\mu_{2}^{\prime}$. For the special case when $v_{1}=v_{2}$, the excess chemical potentials are equal (i.e., $\tilde{\mu}_{1}=\tilde{\mu}_{2}$ ) and drop out of Eq. 10 which then implies that the old variables $\mu_{1}$ and $\mu_{2}$ equalize. But in the case when $v_{1} \neq v_{2}$, the new variable $\mu_{\alpha}^{\prime}$ takes the role of chemical potential which then equalize upon contact. This identification of $\mu_{\alpha}^{\prime}$ as an intensive variable which equalizes for two systems in contact was missed in [11, 14] where it was concluded that there is no such equalization when $v_{1} \neq v_{2}$. Moreover, in contrary to the suggestion in [14], the detailed balance condition is still satisfied at the contact even when $v_{1} \neq v_{2}$.

For a class of systems specified by a particular set of parameters $\left\{f_{\alpha}(n), v_{\alpha}\right\}$, it can be immediately checked that the zeroth law is indeed satisfied. In Fig. 13, we perform various numerical experiments similar to those discussed in Fig. 3 and 4 . We choose $f_{\alpha}(n)=n^{\delta_{\alpha}-1}$ with various sets of parameter values for $\left\{\delta_{\alpha}, v_{\alpha}\right\}$. In the first experiment, ring 1 with $\delta_{1}=3, v_{1}=1$ and density $n_{1} \simeq 2.3$ equilibrated with ring 3 with $\delta_{2}=5$, $v_{2}=0.5$ and density $n_{3} \simeq 7.7$. In the second experiment, ring 2 with $\delta_{1}=4, v_{1}=0.75$ and density $n_{2} \simeq 3.8$ is equilibrated with ring 3 with $\delta_{2}=5, v_{2}=0.5$ and density $n_{3} \simeq 7.7$. In the third experiment, ring 1 with $\delta_{1}=3, v_{1}=1$ and density $n_{1}^{\prime} \simeq 2.3$ is equilibrated with ring 2 with $\delta_{2}=4, v_{2}=0.75$ and density $n_{2}^{\prime} \simeq 3.8$ where $n_{1}^{\prime} \simeq n_{1}$ and $n_{2}^{\prime} \simeq n_{2}$. In this case one can see that the zeroth law is satisfied (see the left panel of Fig. 131). We have also checked that values of the densities are such that chemical potential $\mu_{\alpha}^{\prime}$ takes equal values for two rings in contact. In the right panel of Fig. 13 we again perform three similar numerical experiments but now, in the second experiment, the values of the factors $v_{1}=0.85$ and $v_{2}=0.4$ are slightly perturbed by an arbitrary amount from the earlier values of the factors $v_{1}=0.75$ and $v_{2}=0.5$ which were chosen in the second experiment of the first set of numerical experiments (i.e., corresponding to the left panel of Fig. 13). One can see that, in this case, the zeroth law is violated even though 

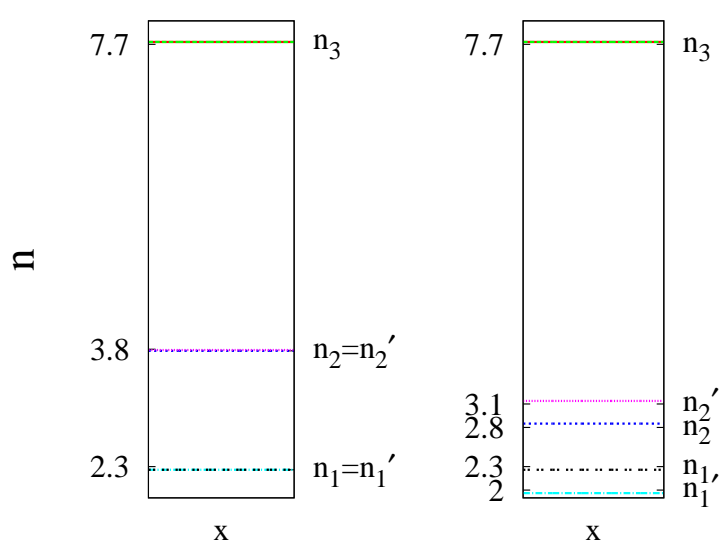

FIG. 13. Density profiles for rings 1, 2 and 3 (all with same size $L=100)$. Left panel: Three numerical experiments to test the zeroth law in the case of the ZRP. Experiment 1: Ring $1\left(\delta_{1}=3, v_{1}=1\right)$ in contact with $\operatorname{ring} 3\left(\delta_{2}=5, v_{2}=0.5\right)$. Experiment 2: Ring $2\left(\delta_{1}=4, v_{1}=0.75\right)$ in contact with ring $3\left(\delta_{2}=5, v_{2}=0.5\right)$. Experiment 3: Ring $1\left(\delta_{1}=3\right.$, $\left.v_{1}=1\right)$ in contact with ring $2\left(\delta_{2}=4, v_{2}=0.75\right)$. The zeroth law is satisfied in this case. Right panel: Three numerical experiments to illustrate the role of contact dynamics for the deviations from the zeroth law. Experiment 1: Ring $1\left(\delta_{1}=3\right.$, $\left.v_{1}=1\right)$ in contact with ring $3\left(\delta_{2}=5, v_{2}=0.5\right)$. Experiment 2 with slightly perturbed jump rates at the contact: Ring 2 $\left(\delta_{1}=4, v_{1}=0.85\right)$ in contact with ring $3\left(\delta_{2}=5, v_{2}=0.4\right)$. Experiment 3: Ring $1\left(\delta_{1}=3, v_{1}=1\right)$ in contact with ring $2\left(\delta_{2}=4, v_{2}=0.75\right)$. The zeroth law is not satisfied in this case.

the factorization property as given in Eq. 8 exactly holds in each of the three experiments. This illustrates the role of the contact dynamics for the zeroth law which is satisfied only for a precise set of jump rates at the contact with no arbitrariness is allowed in these rates.

\section{B. Excess Chemical Potential in the Equilibrium KLS Model}

The above situation in the case of the zero range process (ZRP) is very similar to the special case of the equilibrium KLS model when one chooses the transition rates as given in Eqs. 2 and 3 with no driving in the bulk of the individual systems, i.e., $E_{1}=E_{2}=0$, and a slightly modified transition rates at the contact as follows. In general, the transition rate for a particle jumping from system 1 to system 2 is given by

$$
w\left(C \rightarrow C^{\prime}\right)=v_{1} \times \min \left\{1, e^{-\Delta(0)}\right\}
$$

and the reverse transition rate for the particle jumping from system 2 to system 1 is given by

$$
w\left(C^{\prime} \rightarrow C\right)=v_{2} \times \min \left\{1, e^{-\Delta(0)}\right\}
$$

where we put $\beta=1$. For $v_{1}=v_{2}=1$, the jump rates at the contact are same as given in Eq. 3. Clearly the modified transition rates at the contact amount to an additional field $E_{12}=\ln \left(v_{1} / v_{2}\right)$, say from system 1 to system 2, along the bonds connecting the two systems. Note that the field $E_{12}$ is not a driving field and just introduces an extra overall shift in the chemical potential of the system 1 . The field $E_{12}$ accordingly modifies the energy function from $H$ to $H^{\prime}$ by introducing an extra chemical potential in Eq. 1 i.e., $H^{\prime}=H+E_{12} N_{1}$. In this case, the detailed balance is satisfied with respect to the Boltzmann distribution with the modified energy function $H^{\prime}$. Consequently, one could effectively think of an excess contribution to the equilibrium free energy of the system 1 due to the shift in the chemical potential of the system 1 by an amount $E_{12}$. Now the condition of minimization of the total free energy $F=F_{1}+F_{2}$, as given in Eq. 10 for the zero range process, would imply that the new intensive variables $\mu_{\alpha}^{\prime}=\left(-\partial \ln Z_{\alpha} / \partial N_{\alpha}+\ln v_{\alpha}\right)$, not the variables $\mu_{\alpha}=-\partial \ln Z_{\alpha} / \partial N_{\alpha}$, equalize where free energies of the respective systems are $F_{1}=-\ln Z_{1}+\tilde{\mu}_{1} N_{1}$ and $F_{2}=-\ln Z_{2}+\tilde{\mu}_{2} N_{2}$ with partition function $Z_{\alpha}=$ $\sum_{C} e^{-H_{\alpha}(C)}$, the energy function for individual system $H_{\alpha}=K_{\alpha} \sum_{\left\langle\mathbf{r}_{\alpha}, \mathbf{r}_{\alpha}^{\prime}\right\rangle} \eta\left(\mathbf{r}_{\alpha}\right) \eta\left(\mathbf{r}_{\alpha}^{\prime}\right)$ (see Eq. 10 for $\alpha=1,2$. Consequently, the zeroth law is satisfied in the case when each system is assigned a particular set of values of the factors $v_{\alpha}$. However, if the factors $v_{\alpha}$ are chosen arbitrarily in any of the numerical experiments as discussed in the right panel of Fig. 13 in the case of the ZRP, the zeroth law would not hold even for the equilibrium KLS model with the modified jump rates at the contact.

\section{ANALYTICAL APPROACHES FOR THE DRIVEN KLS MODEL}

In this section, first we discuss how the zeroth law, which has been seen to be satisfied to a very good approximation in the simulations of the driven KLS model for various parameter values, could be explained in terms of large deviation principle. Then we discuss how the deviations from the zeroth law, also observed in the simulations, could be explained by modifying this large deviation principle.

\section{A. Large Deviation Principle}

In equilibrium, the zeroth law can be derived from variational principles, e.g., by maximization of entropy of an isolated system or by minimization of free energy in the case when the system is in contact with a reservoir. For some nonequilibrium systems, there may be a similar principle, called the large deviation principle 2, 24]. Then, the zeroth law can be derived from the large deviation principle along the same line as in equi- 
librium in the following way. Let us consider the following scenario. Two systems are kept in contact with each other with a particular dynamics specified at the contact. The two systems exchange according to the contact dynamics some conserved quantity, say number of particles, such that $N_{1}+N_{2}=N=$ constant with $N_{1}, N_{2}$ being the number of particles in systems 1 and 2 respectively (schematically shown in Fig. 11). The quantities $N_{1}, N_{2}$ are considered to be extensive, i.e., proportional to the volume $V_{1}, V_{2}$ of system 1 and 2 respectively. Now we are interested in large deviations of $N_{1}$ and $N_{2}$ and assume that the probability of large deviation $P\left(N_{1}, N_{2}\right)$ in the quantity $N_{1}$ and $N_{2}$ is given by

$$
P\left(N_{1}, N_{2}\right) \sim \frac{e^{-V_{1} f_{1}\left(n_{1}\right)} e^{-V_{2} f_{2}\left(n_{2}\right)}}{e^{-F(N)}}
$$

in the limit of $N_{1}, N_{2}, V_{1}, V_{2} \gg 1$ keeping respective densities $n_{1}=N_{1} / V_{1}$ and $n_{2}=N_{2} / V_{2}$ finite with the normalization constant $\exp [-F(N)]$. The above equation is the statement of the large deviation principle [2, 24] and the functions $f_{1}\left(n_{1}\right), f_{2}\left(n_{2}\right)$ are called the large deviation functions for the corresponding systems. The sign ' $\sim$ ' implies equality in terms of logarithm of the respective quantities and Eq13 can be written more rigorously as

$$
\begin{array}{r}
-\ln P\left(N_{1}, N_{2}\right)=V_{1} f_{1}\left(n_{1}\right)+V_{2} f_{2}\left(n_{2}\right)-F(N) \\
+\epsilon\left(N_{1}, N_{2}\right)
\end{array}
$$

where $\epsilon\left(N_{1}, N_{2}\right) / \ln P\left(N_{1}, N_{2}\right) \rightarrow 0$ as $N_{1}, N_{2} \rightarrow \infty$. Note that, in writing Eqs. 13 and 14, we have assumed that the correlation between system 1 and 2 can be neglected as a boundary-effect in the limit of large volume. The macroscopic stationary state $\left\{N_{1}^{*}, N_{2}^{*}\right\}$, under the constraint $N_{1}+N_{2}=$ constant, can be determined by maximizing $\ln P\left(N_{1}, N_{2}\right)$, i.e., $\partial \ln P\left(N_{1}, N_{2}\right) / \partial N_{1}=0$ which gives

$$
\left(\frac{\partial f_{1}}{\partial n_{1}}\right)_{n_{1}^{*}}=\left(\frac{\partial f_{2}}{\partial n_{2}}\right)_{n_{2}^{*}}=\mu
$$

where $n_{1}^{*}=N_{1}^{*} / V_{1}, n_{2}^{*}=N_{2}^{*} / V_{2}$ and $\mu$ is the chemical potential which takes same values in the steady state when system 1 and 2 are kept in contact. Note that the validity of Eq. 15, which implies the existence of an intensive thermodynamic variable, follows from the assumption of a large deviation principle of Eq. 13. The above arguments are quite general and could be valid irrespective of a specific nature of the dynamics one considers in a particular problem. Therefore Eq. 15] can be equally applicable to an equilibrium as well as a nonequilibrium steady state. Another consequence of equalization of intensive variables is the fluctuation-response relation between the compressibility and the fluctuations in particle-number $N_{1}$ of system 1, i.e.,

$$
\frac{\partial\left\langle N_{1}\right\rangle}{\partial \mu}=\left\langle N_{1}^{2}\right\rangle-\left\langle N_{1}\right\rangle^{2}
$$

when system 1 is kept in contact with a very large system 2 , with chemical potential $\mu$, which can be considered as a particle reservoir (i.e., $N_{1} \ll N_{2}$ and $V_{1} \ll V_{2}$ ). For more details regarding the above fluctuation relation in the context of the ZRP as well as for the KLS model, see Appendix B.

\section{B. Modified Large Deviation Principle}

The exact results for the ZRP and the equilibrium KLS model give us an insight into the role of the contact dynamics which can effectively introduce an excess chemical potential across the contact region. Consequently, the intensive variable for a system which equalizes upon contact can have different functional forms depending on the specifics of the dynamics at the contact and therefore on the other system in contact. To describe this situation in a more general context where there may be equalization of an intensive variable but still the zeroth law does not strictly hold, we write the large deviation probabilities as given in Eq. 13 in a modified asymptotic form

$$
P\left(N_{1}, N_{2}\right) \sim \frac{e^{-V_{1} f_{1}\left(n_{1}\right)} e^{-V_{2} f_{2}\left(n_{2}\right)} e^{\tilde{\mu}_{1} N_{1}} e^{\tilde{\mu}_{2} N_{2}}}{e^{-F(N)}}
$$

where $f_{1}, f_{2}$ are the large deviation functions for a putative contact dynamics for which the zeroth law is satisfied, the two additional quantities $\tilde{\mu}_{1}, \tilde{\mu}_{2}$ can be thought of as excess chemical potentials arising solely due to the actual contact dynamics which is under consideration and for which the zeroth law is not satisfied. Clearly, for an arbitrarily chosen contact dynamics, the old intensive variables $\mu_{1}=\partial f_{1} / \partial n_{1}$ and $\mu_{2}=\partial f_{2} / \partial n_{2}$ do not equalize. Note that, unlike in the case of the ZRP or the equilibrium KLS model, the potentials $\tilde{\mu}_{1}$ and $\tilde{\mu}_{2}$ is in general a-priori unknown for a given contact dynamics. Moreover, for an arbitrarily chosen contact dynamics, it may not always be possible to assign, to the individual systems, an intensive variable which is independent of the specifics at the contact between two systems. In this case, even though there can be equalization of some intensive variable when two systems are kept in contact, the zeroth law may not be satisfied as demonstrated in the case of the ZRP (see right panel of Fig. 13).

The above modified large deviation principle in Eq. 17 still assumes that two systems in contact have an asymptotic factorization property in the sense that the correlations between the systems can be ignored in the large volume limit. In a special case (e.g., the ZRP or the equilibrium KLS model), the chemical potentials $\tilde{\mu}_{1}$ and $\tilde{\mu}_{2}$ can be constant over an entire density range. However, in general, the quantities $\tilde{\mu}_{1}\left(n_{1}\right)$ and $\tilde{\mu}_{2}\left(n_{2}\right)$ can be functions of densities $n_{1}$ and $n_{2}$. Note that, although the large deviation principles in Eqs. 13 and 17 appear to have different forms, they are essentially the same. The only difference is that, in the modified form of Eq. 17, we 
identify the contribution to the large deviation function due to the contact dynamics and separate this contribution from that arising due to the bulk of the individual systems.

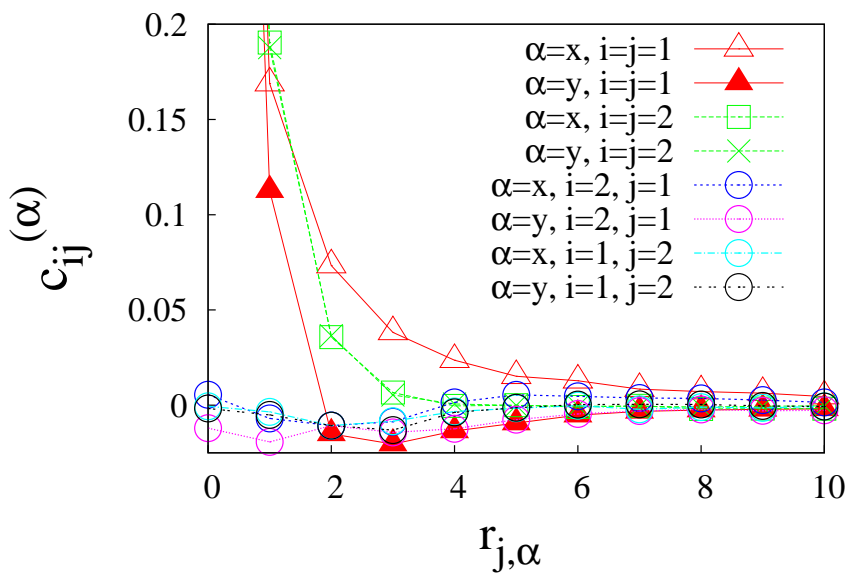

FIG. 14. Correlations between various neighboring sites as a function of distance when a driven system $1(K=-0.75$, $E=4, V=50 \times 50)$ and an equilibrium system $2(K=$ $-0.75, E=0, V=50 \times 50)$ in contact with each other. The function $c_{i j}^{(\alpha)}\left(r_{j, \alpha}\right)$ denotes the scaled correlation (see Eq. 18) along $\alpha$ th direction between two points located in system $i$ and system $j$ where $r_{j, \alpha}$ being the $\alpha$ th component of relative position vector $\mathbf{r}_{\mathbf{j}}$ between the two points with $i, j=1,2$ and $\alpha=x, y$ (for two dimensional system), $\Delta \eta(\mathbf{r})=\eta(\mathbf{r})-$ $\langle\eta(\mathbf{r})\rangle$ the fluctuation in occupation variable $\eta(\mathbf{r})$. For $i \neq j$, $c_{i j}^{(\alpha)}\left(r_{j, \alpha}=0\right)$ is the correlation between two nearest-neighbor sites located across the contact.

The asymptotic factorization can indeed be a very good approximation even in the case of a more complicated driven lattice gas like the KLS model. To show this, we now study various spatial density-correlations $c_{i j}^{(\alpha)}\left(r_{j, \alpha}\right)$ between two points located in the individual systems as well as two points located in two different systems across the contact. We define the function

$$
c_{i j}^{(\alpha)}\left(r_{j, \alpha}\right)=\frac{\left\langle\left\{\Delta \eta\left(\mathbf{r}_{\mathbf{i}}^{\mathbf{c}}\right)\right\}\left\{\Delta \eta\left(\mathbf{r}_{\mathbf{j}}^{\mathbf{c}}+\mathbf{r}_{\mathbf{j}}\right)\right\}\right\rangle}{\sqrt{\left\langle\left\{\Delta \eta\left(\mathbf{r}_{\mathbf{i}}^{\mathbf{c}}\right)\right\}^{2}\right\rangle} \sqrt{\left\langle\left\{\Delta \eta\left(\mathbf{r}_{\mathbf{j}}^{\mathbf{c}}+\mathbf{r}_{\mathbf{j}}\right)\right\}^{2}\right\rangle}},
$$

which denotes the scaled correlation along $\alpha$ th direction between two points in system $i$ and system $j$ where $r_{j, \alpha}$ the $\alpha$ th component of position vector $\mathbf{r}_{\mathbf{j}}$ with $i, j=1,2$ and $\alpha=x, y$ (for a two-dimensional system), $\Delta \eta(\mathbf{r})=$ $\eta(\mathbf{r})-\langle\eta(\mathbf{r})\rangle$ the fluctuation in occupation variable $\eta(\mathbf{r})$, $\mathbf{r}_{\mathbf{i}}^{\mathbf{c}}$ the position vector of the contact site in system $i$. For $i=j, c_{i i}^{(\alpha)}\left(r_{i, \alpha}\right)$ denotes density-correlations, along $\alpha$-axis, between two points both located in the same system $i$ and, for $i \neq j, c_{i j}^{(\alpha)}\left(r_{j, \alpha}\right)$ denotes cross-correlation, along $\alpha$-axis, between two points one located in the system $i$ and the other located in system $j$. In Fig. 14, we have plotted the scaled correlation $c_{i j}^{(\alpha)}\left(r_{j, \alpha}\right)$ as a function of $r_{j, \alpha}$ for a driven system with $K_{1}=-0.75$, $E_{1}=4$ and an equilibrium system with $K_{2}=-0.75$ where the systems are kept in contact, both at density $n=1 / 2$. One can see that the amplitude of the cross-correlations among nearest-neighbor sites located in two different systems across the contact are very small, almost an order of magnitude smaller as compared to those among neighboring sites in the individual systems, i.e., $c_{21}^{(\alpha)}(0), c_{12}^{(\alpha)}(0) \ll c_{11}^{(\alpha)}(1), c_{22}^{(\alpha)}(1)$. Therefore the asymptotic factorization property is expected to be wellsatisfied in this case. Moreover, the very weak crosscorrelation between two systems in contact explains why the density profiles remain almost homogeneous even around the contact. Note that the density-correlation functions $c_{11}^{(x)}\left(r_{1, x}\right)$ and $c_{11}^{(y)}\left(r_{1, y}\right)$ for the driven system along $x$ and $y$ directions, respectively, are clearly different due to the presence of a strong driving field which breaks the isotropy. Whereas, for the equilibrium system, correlations $c_{22}^{(x)}\left(r_{1, x}\right)$ and $c_{22}^{(y)}\left(r_{1, y}\right)$ expectedly remain the same. For attractive interaction strengths, the correlations perpendicular to the driving field become negative at larger distances. This picture remains qualitatively the same at other densities as well.

\section{Excess Chemical Potential in the Driven KLS Model}

The role of the contact dynamics in the KLS model can be more complex than that in the previously discussed models of the ZRP. However, interestingly for some parameter values, we indeed find evidence of a constant difference $\delta \mu=\tilde{\mu}_{1}-\tilde{\mu}_{2}$ in the excess chemical potentials in a wide density range even in the KLS model for nonzero driving. This supports the modified form of the large deviation principle even for the KLS model.

We consider density $n$ as a function of chemical potential $\mu$ for a driven system with $K=-1, E=6$ which is separately kept in contact with various equilibrium systems with interaction strengths $K=0, K=-0.8$, and $K=-0.9$. As we have already discussed in Fig. 11 for the same systems, there were indeed deviations from the zeroth law observed as the different curves for $n v s$. $\mu$ in Fig. 11 do not fall on each other. However, now by shifting chemical potential $\mu$ to $\mu+\delta \mu$ and choosing a suitable $\delta \mu$ in each of the cases, all the curves can be made to collapse on each other quite well as can be seen in Fig. 15 .

We have done the same analysis for another driven system with a different set of parameter values $K=3.75$, $E=6$. The driven system is separately kept in contact with two equilibrium systems with interaction strengths $K=0.75$ and $K=1.0$. In Fig. 16, the densities $n$ are plotted as a function of the shifted chemical potential $\mu$ (also see Fig. 12). The curves could be collapsed on top 


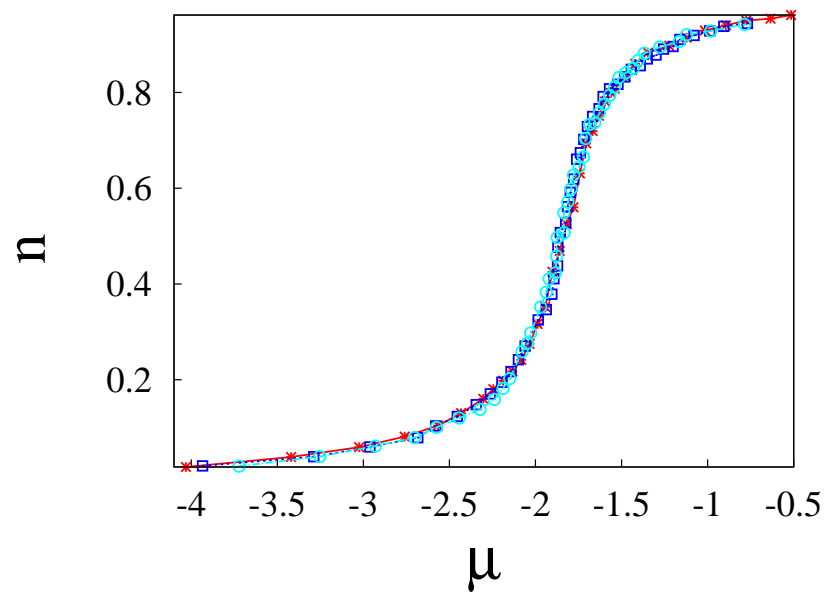

FIG. 15. Density $n$ vs. shifted chemical potential $\mu$ is plotted for a driven system with $K=-1, E=6$ which is separately kept in contact with three equilibrium systems with interaction strengths $K=0, K=-0.8$, and $K=-0.9$. By shifting $\mu \rightarrow \mu+\delta \mu$, all curves collapse on each other well where we choose $\delta \mu=0.06$ and $\delta \mu=0.095$ for the cases when the driven system is in contact with the equilibrium systems with $K=-0.8$ and $K=-0.9$, respectively.

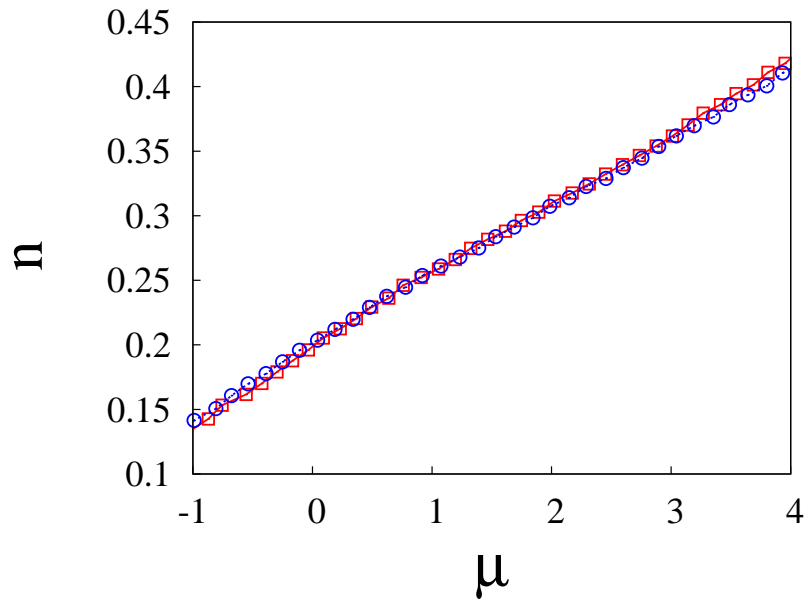

FIG. 16. Density $n$ vs. shifted chemical potential $\mu$ is plotted for a driven system with $K=3.75, E=6$ which is separately kept in contact with two equilibrium systems with $K=0.75$ and $K=1$. Shifting $\mu \rightarrow \mu+\delta \mu$, two curves collapse on each other reasonably well by choosing $\delta \mu=0.1$ for the case when the driven system is in contact with the equilibrium system with $K=0.75$.

of each other reasonably well by shifting the $\mu$ to $\mu+\delta \mu$.

However, in both the cases of Figs. 15] and 16, it should be noted that the collapse is not so good at very low chemical potentials (i.e., low densities). This indicates that the difference in the excess chemical potential $\delta \mu$ actually may not be constant over the entire chemical potential range and can depend on the chemical potential itself (or equivalently density) of the corresponding equilibrium system in contact.

\section{CONCLUDING PERSPECTIVE}

In summary, in this paper we have studied "equilibration" of driven lattice gases when two systems are kept in contact and allowed to exchange particles with the total number of particles conserved. Interestingly, both for attractive as well as for repulsive nearest-neighbor interactions and for a wide range of parameter values, there is a remarkably simple, though approximate, thermodynamic structure where the zeroth law is quite well satisfied.

However, there are also observed deviations from this simple thermodynamic law. To understand these deviations, first we have studied the nontrivial role of the contact dynamics for a variant of the zero range process (ZRP) as well as a variant of the equilibrium KatzLebowitz-Spohn (KLS) model, where one can calculate the steady-state probability distribution exactly. Using these simple examples, we point out that, due to the modified contact dynamics, there can be an excess chemical potential induced across the contact region. These results lead us to express the large deviation principle in a modified form which elucidates the role of the contact and which can substantially account for the deviations from the zeroth law.

It is important to note that the modified form of the large deviation principle is still based on the asymptotically factorized form of the steady-state distribution for two driven systems in contact and is valid if the correlations between the systems can be neglected in the large volume limit. In the case of the variant of the ZRP discussed here, these correlations are zero. In the case of the driven KLS model, we observed that the spatial density-correlations across the contact are indeed very small compared to the nearest-neighbor correlations in the individual systems. Interestingly, we found evidence of an almost constant excess chemical potential for various parameter values and in a wide range of densities, therefore supporting applicability of the modified large deviation principle to the KLS model.

In general, the results in this paper lead us roughly to the following possible scenarios for driven systems in contact.

(1) The large deviation principle, or in other words the asymptotic factorization property, may break down due to long-ranged correlations which may be present in the driven systems [25 27]. In this case, the combined system cannot be divided into independent subsystems and there would be no intensive variable which equalizes upon contact.

(2) However, when the amplitude of these correlations are sufficiently weak, it is possible that a large deviation 
principle holds, although in a modified form. In these cases, the systems can be characterized by the excess chemical potentials across the contact and consequently there would be some intensive variable which would then equalize upon contact. Note that introducing the excess chemical potential is essentially a way of reparametrization of the chemical potential of the driven system under consideration. These excess chemical potentials can depend on the specifics of the contact dynamics and they are generally a-priori unknown, therefore in a sense arbitrary. For some parameter values, the excess chemical potential may almost be constant over a range of densities. In special cases, the arbitrariness of the excess chemical potential can be removed by choosing a suitable contact dynamics such that the zeroth law holds strictly. In these cases, it is actually possible to assign to the individual systems an intensive variable, independent of the specifics at the contact between two systems, which would then equalize. It should be noted here that the modified large deviation principle could be satisfied irrespective of whether the zeroth law is satisfied or not, which has been illustrated in this paper by using a variant of the ZRP.

For the ZRP, since the steady state properties are exactly known, it is easier to choose a contact dynamics so that the zeroth law can be made to be satisfied. However, for the KLS model which has nontrivial steady state properties, it would be difficult to find a contact dynamics, even if it exists, for which the zeroth law would hold strictly. For the KLS model, here we have considered a contact dynamics, mainly based on a physical ground albeit on an ad-hoc basis, which satisfies the local detailed balance condition. The simple modification of the large deviation principle suggested in this paper indicates that it could still be possible to choose a contact dynamics such that the thermodynamic laws are satisfied even better. As an open question, the origin of the excess chemical potential at the contact should be understood in more detail which could give valuable insights whether it is possible to choose a contact dynamics for which a simple thermodynamic structure emerges for driven systems in general.

There are further important aspects in exploring such a simple structure for driven systems in contact. Unlike in equilibrium where there is a well defined prescription to describe various thermodynamic properties using the standard Boltzmann distribution, there is no such prescription for nonequilibrium systems. However, the numerically observed simple thermodynamic structure concerning driven lattice gases in contact may give us an useful tool to characterize such systems and gives rise to a possibility to describe phase transitions which are known to occur in these driven interacting many-particle systems.

\section{ACKNOWLEDGMENTS}

We thank R. K. P. Zia for stimulating discussions.

\section{APPENDIX A: STEADY STATE DISTRIBUTION FOR THE ZRP WHEN $v_{1} \neq v_{2}$}

In the general case when $v_{1} \equiv v_{1}^{(c)} \neq v_{2} \equiv v_{2}^{(c)}$, the ansatz for the steady-state probability distribution is given by

$$
\begin{aligned}
P_{s t}\left(\left\{n_{i_{1}}\right\},\left\{n_{i_{2}}\right\}\right)= & \frac{1}{Z_{N}}\left[\prod_{i_{1}=1}^{L_{1}} f_{1}\left(n_{i_{1}}\right) \prod_{i_{2}=1}^{L_{2}} f_{2}\left(n_{i_{2}}\right)\right] \\
& \times \frac{1}{v_{1}^{N_{1}} v_{2}^{N_{2}}} \delta\left(N_{1}+N_{2}-N\right)
\end{aligned}
$$

where $N_{1}$ and $N_{2}$ are number of particles in ring 1 and ring 2 respectively and $N=N_{1}+N_{2}$ is the total conserved particle number. Now we consider the following two cases - (1) when particle jumps in the bulk, say in ring 1 , and (2) when particle jumps from one ring to the other.

Case (1): Consider the following two transitions from a configuration $C$ to $C^{\prime}$ and a configuration $C^{\prime \prime}$ to $C$ where $C \equiv\left(\left\{\ldots, n_{i_{1}-1}, n_{i_{1}}, n_{i_{1}+1}, \ldots\right\},\left\{n_{i_{2}}\right\}\right)$, $C^{\prime} \equiv\left(\left\{\ldots, n_{i_{1}-1}, n_{i_{1}}-1, n_{i_{1}+1}+1, \ldots\right\},\left\{n_{i_{2}}\right\}\right)$ and $C^{\prime \prime} \equiv$ $\left(\left\{\ldots, n_{i_{1}-1}+1, n_{i_{1}}-1, n_{i_{1}+1}, \ldots\right\},\left\{n_{i_{2}}\right\}\right)$. The steadystate probability current $J\left(C \rightarrow C^{\prime}\right)=P_{s t}(C) w\left(C^{\prime} \mid C\right)$ from configuration $C$ to $C^{\prime}, P_{s t}(C)$ being the probability of configuration $C$ in steady state and $w\left(C^{\prime} \mid C\right)$ being the transition rate from $C$ to $C^{\prime}$, can be explicitly written as

$$
\begin{array}{r}
J\left(C \rightarrow C^{\prime}\right)=\frac{1}{Z_{N}}\left[\ldots f\left(n_{i_{1}-1}\right) f\left(n_{i_{1}}\right) f\left(n_{i_{1}+1}\right) \ldots\right] \\
\times\left[v_{1}^{(b)} \frac{f\left(n_{i_{1}}-1\right)}{f\left(n_{i_{1}}\right)}\right]\left[\prod_{i_{2}=1}^{L_{2}} f_{2}\left(n_{i_{2}}\right)\right] \\
\times \frac{1}{v_{1}^{N_{1}} v_{2}^{N_{2}}} \delta\left(N_{1}+N_{2}-N\right)
\end{array}
$$

where we have used jump rate in the bulk of ring 1 which is $w\left(C^{\prime} \mid C\right)=v_{1}^{(b)} f\left(n_{i_{1}}-1\right) / f\left(n_{i_{1}}\right)$. Finally we get

$$
\begin{aligned}
J(C \rightarrow & \left.C^{\prime}\right)=\frac{1}{Z_{N}}\left[\ldots f\left(n_{i_{1}-1}\right) f\left(n_{i_{1}}-1\right) f\left(n_{i_{1}+1}\right) \ldots\right] \\
\times & {\left[\prod_{i_{2}=1}^{L_{2}} f_{2}\left(n_{i_{2}}\right)\right] \frac{v_{1}^{(b)}}{v_{1}^{N_{1}} v_{2}^{N_{2}}} \delta\left(N_{1}+N_{2}-N\right) . }
\end{aligned}
$$

Similarly the probability current $J\left(C^{\prime \prime} \rightarrow C\right)=$ $P\left(C^{\prime \prime}\right) w\left(C \mid C^{\prime \prime}\right)$ from configuration $C^{\prime \prime}$ to $C$ can be explicitly written as

$J\left(C^{\prime \prime} \rightarrow C\right)=\frac{1}{Z_{N}}\left[\ldots f\left(n_{i_{1}-1}+1\right) f\left(n_{i_{1}}-1\right) f\left(n_{i_{1}+1}\right) \ldots\right]$ 


$$
\begin{array}{r}
\times\left[v_{1}^{(b)} \frac{f\left(n_{i_{1}-1}\right)}{f\left(n_{i_{1}-1}+1\right)}\right]\left[\prod_{i_{2}=1}^{L_{2}} f_{2}\left(n_{i_{2}}\right)\right] \\
\times \frac{1}{v_{1}^{N_{1}} v_{2}^{N_{2}}} \delta\left(N_{1}+N_{2}-N\right) \\
=\frac{1}{Z_{N}}\left[\ldots f\left(n_{i_{1}-1}\right) f\left(n_{i_{1}}-1\right) f\left(n_{i_{1}+1}\right) \ldots\right] \\
\times\left[\prod_{i_{2}=1}^{L_{2}} f_{2}\left(n_{i_{2}}\right)\right] \frac{v_{1}^{(b)}}{v_{1}^{N_{1}} v_{2}^{N_{2}}} \delta\left(N_{1}+N_{2}-N\right) . \quad(22)
\end{array}
$$

Clearly the probability currents $J\left(C \rightarrow C^{\prime}\right)$ and $J\left(C^{\prime \prime} \rightarrow C\right)$ are equal. Since, for any transition from $C$ to $C^{\prime}$, it is always possible to find a corresponding transition $C^{\prime \prime}$ to $C$, the net current into $C$ vanishes pairwise, i.e., $J\left(C \rightarrow C^{\prime}\right)=J\left(C^{\prime \prime} \rightarrow C\right)$ in the steady state.

Case (2): Consider two transitions from $C$ to $C^{\prime}$ and the configuration $C^{\prime}$ to $C$ where $C \equiv\left(\left\{\ldots, n_{k_{1}}, \ldots\right\},\left\{\ldots, n_{k_{2}}, \ldots\right\}\right), \quad C^{\prime} \equiv$ $\left(\left\{\ldots, n_{k_{1}}-1, \ldots\right\},\left\{\ldots, n_{k_{2}}+1, \ldots\right\}\right)$, and $k_{1}$ and $k_{2}$ are respective contact sites in ring 1 and ring 2 . In this case the probability current $J\left(C \rightarrow C^{\prime}\right)$ can be written as

$$
\begin{array}{r}
J\left(C \rightarrow C^{\prime}\right)=\frac{1}{Z_{N}}\left[\ldots f\left(n_{k_{1}}\right) \ldots\right]\left[v_{1} \frac{f\left(n_{k_{1}}-1\right)}{f\left(n_{k_{1}}\right)}\right] \\
\times\left[\ldots f\left(n_{k_{2}}\right) \ldots\right] \frac{1}{v_{1}^{N_{1}} v_{2}^{N_{2}}} \delta\left(N_{1}+N_{2}-N\right) \\
=\frac{1}{Z_{N}}\left[\ldots f\left(n_{k_{1}}-1\right) \ldots\right]\left[\ldots f\left(n_{k_{2}}\right) \ldots\right] \\
\times \frac{1}{v_{1}^{N_{1}-1} v_{2}^{N_{2}}} \delta\left(N_{1}+N_{2}-N\right)
\end{array}
$$

Similarly the probability current $J\left(C^{\prime} \rightarrow C\right)$ can be written as

$$
\begin{array}{r}
J\left(C^{\prime} \rightarrow C\right)=\frac{1}{Z_{N}}\left[\ldots f\left(n_{k_{1}}-1\right) \ldots\right]\left[\ldots f\left(n_{k_{2}}+1\right) \ldots\right] \\
\times\left[v_{2} \frac{f\left(n_{k_{2}}\right)}{f\left(n_{k_{2}}+1\right)}\right] \frac{1}{v_{1}^{N_{1}-1} v_{2}^{N_{2}+1}} \delta\left(N_{1}+N_{2}-N\right) \\
=\frac{1}{Z_{N}}\left[\ldots f\left(n_{k_{1}}-1\right) \ldots\right]\left[\ldots f\left(n_{k_{2}}\right) \ldots\right] \\
\times \frac{1}{v_{1}^{N_{1}-1} v_{2}^{N_{2}}} \delta\left(N_{1}+N_{2}-N\right) .
\end{array}
$$

Clearly the net probability current into $C$ is again zero as $J\left(C \rightarrow C^{\prime}\right)=J\left(C^{\prime} \rightarrow C\right)$ in the steady state. This completes the proof for the steady-state ansatz given in Eq. 19 which satisfies Master equation $\partial_{t} P_{s t}(C, t)=0=$ $\sum_{C^{\prime} \neq C}\left[P_{s t}\left(C^{\prime}\right) w\left(C^{\prime} \rightarrow C\right)-P_{s t}(C) w\left(C \rightarrow C^{\prime}\right)\right]$. Note that the condition $J\left(C \rightarrow C^{\prime}\right)=J\left(C^{\prime} \rightarrow C\right)$ is nothing but the detailed balance condition which is satisfied at the contact even when $v_{1} \neq v_{2}$. However, detailed balance is not satisfied in the bulk and consequently there are nonzero currents within the individual systems.

\section{APPENDIX B: FLUCTUATION-RESPONSE RELATIONS}

One interesting consequence of Eq. 15 is a relation between the susceptibility and the fluctuation in particlenumber of a system when the system is in contact with a large reservoir characterized by a chemical potential $\mu$. Consider system 1 is in contact with system 2 which is very large compared to system 1 . Let us denote $\sigma_{N_{1}}^{2}$ as the standard deviation of fluctuations in the total number of particles $N_{1}$ in system 1, i.e., $\sigma_{N_{1}}^{2}=\left\langle N_{1}^{2}\right\rangle-\left\langle N_{1}\right\rangle^{2}$. Then the large deviation principle with the definition of chemical potential as given in Eq. 15 implies that the fluctuation of particle-number around an average particle-number $N_{1}^{*}$,

$$
P\left(N_{1}\right) \approx \text { constant } \times e^{-\left(N_{1}-N_{1}^{*}\right)^{2} / 2 \chi}
$$

where $1 / \chi=\partial \mu / \partial N_{1}=\left(\partial^{2} F_{1} / \partial N_{1}^{2}\right)_{N_{1}^{*}}$ with $F_{1}\left(N_{1}\right)=$ $V_{1} f_{1}\left(n_{1}\right)$. Since the root mean square fluctuation in the particle-number $N_{1}$ is $\sigma_{N_{1}}^{2}=2 \chi$, one gets the following fluctuation relation,

$$
\frac{\partial\left\langle N_{1}\right\rangle}{\partial \mu}=\left(\left\langle N_{1}^{2}\right\rangle-\left\langle N_{1}\right\rangle^{2}\right)
$$

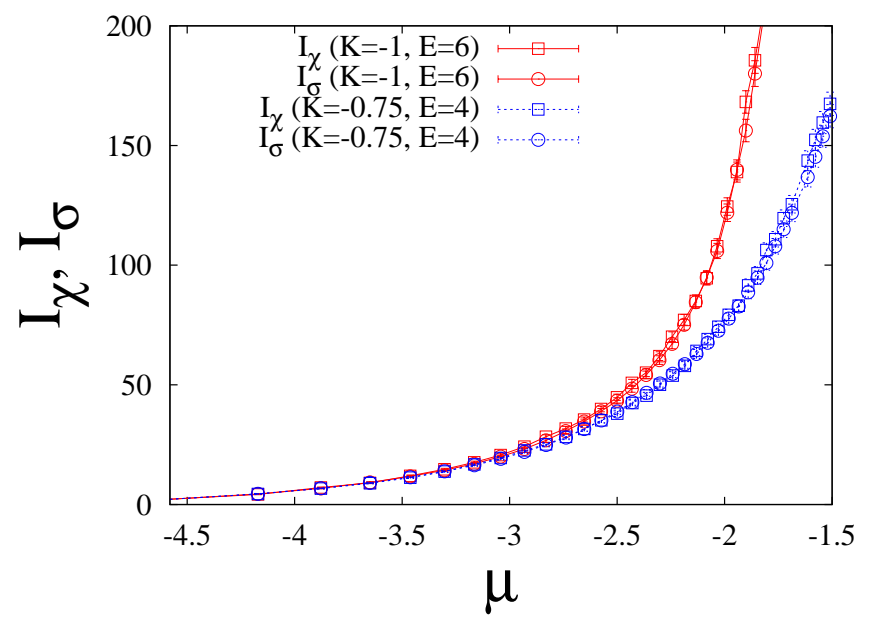

FIG. 17. Driven system in contact with an equilibrium reservoir of noninteracting hardcore particles. Integrated compressibility $I_{\chi}$ (squares) and integrated fluctuation $I_{\sigma}$ (circles) plotted as a function of $\mu$ for a driven system with two different sets of parameter values with $K=-1, E=6$ (red) and $K=-0.75, E=4$ (blue). The fluctuation-response relation is well satisfied.

For the ZRP, even when $v_{1} \neq v_{2}$, the fluctuationresponse relation between the compressibility and the root mean square fluctuation in particle-number $N_{1}$ is still exactly satisfied if the ring 1 is in contact with a much larger ring 2 being a particle reservoir. Moreover, since in this case the variables $\mu_{\alpha}^{\prime}$ and $\mu_{\alpha}$ differ only by 
a constant $\ln v_{\alpha}$ (see Eq. 10 and the paragraph below), the fluctuation-response relation is satisfied with respect to both the new and old intensive variables $\mu_{\alpha}^{\prime}$ and $\mu_{\alpha}$, respectively.

Now we briefly discuss the numerical results concerning the fluctuation-response relation for the KLS model with attractive interaction strengths. To numerically test the fluctuation relation as given in Eq. 26, we consider a driven system which is in contact with an equilibrium reservoir of hardcore particles which are otherwise noninteracting (i.e., $K=0$ ). The chemical potential $\mu$ of the equilibrium hardcore particle-reservoir is given by the expression in Eq. 4. For better numerical accuracy, we check the integrated version of Eq. 26, i.e., we calculate the integrated fluctuation $I_{\sigma}=\int_{\mu_{0}}^{\mu}\left(\sigma_{N_{1}}^{2}\right) d \mu^{\prime}$ and the integrated susceptibility $I_{\chi}=\int_{\mu_{0}}^{\mu}\left(d\left\langle N_{1}\right\rangle / d \mu^{\prime}\right) d \mu^{\prime}$ for different values of $\mu$ obtained by varying density of the equilibrium reservoir. We take a two-dimensional $20 \times 20$ nonequilibrium system in contact with a $250 \times 250$ equilibrium reservoir. In Fig. 17 the integrated compressibility and the integrated fluctuations are plotted as a function of chemical potential $\mu$ for two driven systems with two different sets of parameter values with (1) $K=-1, E=6$ and (2) $K=-0.75, E=4$. In these cases, the fluctuationresponse relation is remarkably well satisfied as seen in Fig. 17.

[1] R. K. P. Zia and B. Schmittmann, J. Stat. Mech. P07012 (2007).

[2] G. L. Eyink, J. L. Lebowitz, and H. Spohn, J. Stat. Phys. 83, 385 (1996).

[3] Y. Oono and M. Paniconi, Prog. Theor. Phys. Suppl. 130, 29 (1998).

[4] L. Bertini, A. D. Sole, D. Gabrielli, G. Jona-Lasinio, and C. Landim, Phys. Rev. Lett. 87, 040601 (2001). L. Bertini, A. D. Sole, D. Gabrielli, G. Jona-Lasinio, and C. Landim, J. Stat. Phys. 107, 635 (2002).

[5] T. Bodineau and B. Derrida, Phys. Rev. Lett. 92, 180601 (2004).
[6] S. Sasa and H. Tasaki, J. Stat. Phys. 125, 125 (2006).

[7] H-Q Wang and N. Menon, Phys. Rev. Lett. 100, 158001 (2008).

[8] S. Henkes, C. S. O'Hern, and B. Chakraborty, Phys. Rev. Lett. 99, 038002 (2007).

[9] Y. Shokef, G. Shulkind, and D. Levine, Phys. Rev. E 76, 030101(R) (2007).

[10] K. Hayashi and S. Sasa, Phys. Rev. E 68, 035104 (2003).

[11] E. Bertin, O. Dauchot, and M. Droz, Phys. Rev. Lett. 96, 120601 (2006).

[12] M. R. Evans and T. Hanney, J. Phys. A 38, R195 (2005).

[13] S. N. Majumdar, M. R. Evans, and R. K. P. Zia, Phys. Rev. Lett. 94, 180601 (2005). M. R. Evans, T. Hanney, and S. N. Majumdar, Phys. Rev. Lett. 97, 010602 (2006).

[14] E. Bertin, K. Martens, O. Dauchot, and M. Droz, Phys Rev. E 75, 031120 (2007).

[15] S. Katz et al., J. Stat. Phys. 34, 497 (1984). S. Katz, J. L. Lebowitz, and H. Spohn, J. Stat. Phys. 34, 497 (1984).

[16] B. Schmittmann and R. K. P. Zia, Phys. Rep. 301, 45 (1998). R. K. P. Zia, J. Stat. Phys. 138, 20 (2010).

[17] W. Dietrich, P. Fulde, and I. Peschel, Adv. Phys. 29, 527 (1980).

[18] J. Marro, J. L. Lebowitz, H. Spohn, and M. H. Kalos, J. Stat. Phys. 38, 725 (1985). J. L. Valles and J. Marro, J. Stat. Phys. 43, 441 (1986). ibid 49, 89 (1987). K.-t Leung, Phys. Rev. Lett. 66, 453 (1991). J-S Wang, J. Stat. Phys. 82, 1409 (1996).

[19] K.-t Leung, B. Schmittmann, and R. K. P. Zia, Phys. Rev. Lett. 62, 1772 (1989).

[20] R. Dickman, Phys. Rev. A 38, 2588 (1988). ibid 41, 2192 (1990).

[21] H. K. Janssen and B. Schmittmann, Z. Phys. B - Cond. Matt. 64, 503 (1986). K.-t Leung and J. L. Cardy, J. Stat. Phys. 44, 567 (1986).

[22] P. Pradhan, C. P. Amann, and U. Seifert, Phys. Rev. Lett. 105, 150601 (2010).

[23] A. Achahbar and J. Marro, J. Stat. Phys. 78, 1493 (1995). C. C. Hill, R. K. P. Zia, and B. Schmittmann, Phys. Rev. Lett. 77, 514 (1996).

[24] H. Touchette, Phys. Rep. 478, 1 (2009).

[25] J. R. Dorfman, T. R. Kirkpatrick, and J. V. Sengers, Annu. Rev. Chem. 45, 213 (1994).

[26] P. L. Garrido et al., Phys. Rev. A 42, 1954 (1990). P. L. Garrido, J. L. Lebowitz, C. Maes, and H. Spohn, Phys. Rev. A 42, 1954 (1990).

[27] G. Grinstein, D. H. Lee, and S. Sachdev, Phys. Rev. Lett. 64, 1927 (1990). 